\title{
A Linear Stability Analysis of Thermal Convection in a Fluid Layer with Simultaneous Rotation and Magnetic Field Acting in Different Directions
}

\author{
Ruben Avila and Ares Cabello \\ Departamento de Termofluidos, Facultad de Ingeniería, Universidad Nacional Autónoma de México, 04510 México, DF, Mexico \\ Correspondence should be addressed to Ruben Avila; ravila@unam.mx
}

Received 11 February 2013; Accepted 22 May 2013

Academic Editor: Zhijun Zhang

Copyright (C) 2013 R. Avila and A. Cabello. This is an open access article distributed under the Creative Commons Attribution License, which permits unrestricted use, distribution, and reproduction in any medium, provided the original work is properly cited.

\begin{abstract}
The onset of thermal convection of a Boussinesq fluid located in an unbounded layer heated from below and subject simultaneously to rotation and magnetic field, whose vectors act in different directions, is presented. To the knowledge of the authors, the convective thermal instability analysis for this complex problem has not been previously reported. In this paper, we use the Tau Chebyshev spectral method to calculate the value of the critical parameters (wave number and Rayleigh number at the onset of convection) as a function of (i) different kinds of boundaries, (ii) angle between the three vectors, and (iii) different values of the Taylor number $T$ (rate of rotation) and magnetic parameter $Q$ (strength of the magnetic force). For the classical problems previously reported in the literature, we compare our calculations with Chandrasekhar's variational method results and show that the present method is applicable.
\end{abstract}

\section{Introduction}

The thermal instability of a horizontal layer of fluid heated from below (Rayleigh-Bénard problem) is an important and classical fluid dynamics problem in science and engineering. The linear stability analysis of this problem, in terms of normal modes, has been carried out and reported in several investigations.

The rotating Rayleigh-Bénard problem has also been widely studied and it has been found that the effect of the Coriolis force due to rotation of the infinite layer of fluid, when the rotation vector $\boldsymbol{\Omega}$ acts about the vertical direction, that is, parallel to the gravity force vector $\mathbf{g}$, inhibits the onset of convection and thus induces a stabilizing effect [1$3]$. The amount of the stabilizing effect depends on the rate of rotation (the Taylor number $T$ ). Results for the problem in which the vectors $\boldsymbol{\Omega}$ and $\mathbf{g}$ act in different directions, with an angle $\vartheta_{R}$ between them, were not explicitly reported by $[1,4]$; instead an analogy with the closely related problem of thermal convection subject to the action of a magnetic field was presented by $[1]$.
Moreover, it has also been reported that, in the magnetic Rayleigh-Bénard problem, a stabilizing effect is obtained when the layer of an electrically conducting fluid is immersed in a uniform magnetic field vector $\mathbf{H}$ that is parallel to the gravity force vector $\mathbf{g}$ [5]. The extent of the inhibition depends on the value of the nondimensional magnetic parameter $Q$. It has been found that when $\mathbf{H}$ and $\mathbf{g}$ are parallel, convection at marginal stability is characterized by a cellular pattern; hence, longitudinal and transverse rolls appear simultaneously. Results for the problem in which the vectors $\mathbf{g}$ and $\mathbf{H}$ act in different directions, with an angle $\vartheta_{H}$ between them, and when instability sets in as stationary convection have been previously obtained by considering two cases: (i) solution of the perturbation equations which are independent of $x_{1}$ and (ii) solutions of the perturbation equations that conduct to a more general patterns of motion. In the former case, the onset of instability is characterized by rolls in the $x_{1}$ direction, whereas in the latter case, by using a variational procedure, [1] shows that as the parameter $C_{H}$, defined as $C_{H}=a_{x} \tan \vartheta_{H}$ (where $a_{x}$ is the wave number in the $x_{1}$ direction), increases, keeping the Chandrasekhar number $Q$ 
TABLE 1: Linear problems under study. $\vartheta_{R}$ is the angle between the rotation vector $\Omega$ and the gravity vector $\mathbf{g}$. If $\vartheta_{R} \neq 0, \Omega$ lies in the $x_{1} x_{3}$-plane. $\vartheta_{H}$ is the angle between the magnetic field vector $\mathbf{H}$ and the gravity vector $\mathbf{g}$. If $\vartheta_{H} \neq 0, \mathbf{H}$ lies in the $x_{1} x_{3}$-plane. $\mathbf{g}$ acts along the $x_{3}$ direction; see Figure 1.

\begin{tabular}{|c|c|c|c|c|c|}
\hline Problem & Gravity, $\mathbf{g}$ & Rotation, $\boldsymbol{\Omega}$ & Magnetic field, $\mathbf{H}$ & $\vartheta_{R}$ & $\overline{\vartheta_{H}}$ \\
\hline 1 (Section 5.1) & Yes & Yes & No & $=0$ & - \\
\hline 2 (Section 5.2) & Yes & Yes & No & $\neq 0$ & - \\
\hline 3 (Section 5.3 ) & Yes & No & Yes & - & $=0$ \\
\hline 4 (Section 5.4$)$ & Yes & No & Yes & - & $\neq 0$ \\
\hline 5 (Section 5.5) & Yes & Yes & Yes & $=0$ & $=0$ \\
\hline $6($ Section 5.6$)$ & Yes & Yes & Yes & $\neq 0$ & $\neq 0$ \\
\hline
\end{tabular}

(defined by $Q=\mu^{2}\left(H \cos \vartheta_{H}\right)^{2} \widehat{D}^{2} \widehat{\sigma} / \rho_{o} \nu$, where $\mu, H, \widehat{D}, \widehat{\sigma}$, $\rho_{o}$, and $\nu$ are the magnetic permeability, the magnitude of the magnetic field, the distance between the two plates, the coefficient of electrical conductivity, the density of reference, and the kinematic viscosity, resp.) as a constant value, both the critical Rayleigh number $R$ and the wave number $a=$ $\sqrt{a_{x}^{2}+a_{y}^{2}}$ (where $a_{y}$ is the wave number in the $x_{2}$ direction) at the onset of stationary convection shift to higher values. It has been shown that when $\mathbf{H}$ is very slightly inclined to the direction of gravity, the extent to which transverse rolls are suppressed at marginal stability is also very slight. According to [1], when longitudinal rolls and transverse rolls appear simultaneously, a cellular pattern of convection emerges. Therefore if $\mathbf{H}$ and $\mathbf{g}$ (as well as $\boldsymbol{\Omega}$ and $\mathbf{g}$ ) are not parallel, convection at marginal stability occurs as longitudinal rolls. On the other hand, when $\mathbf{H}$ is impressed with an inclination angle $\vartheta_{H}=\pi / 2$ to the direction of the vertical and $\mathbf{H}$ lies in the $x_{1} x_{3}$-plane, the vertical component of the magnetic field $\left(H \cos \vartheta_{H}\right)$ is equal to zero. The authors in [6] found that a magnetic field, impressed in a horizontal direction $\left(\vartheta_{H}=\pi / 2\right)$, did not inhibit convection, even though the magnetic field was five times stronger than that one needed to suppress convection when acting in the vertical direction $\left(\vartheta_{H}=0\right)$; hence, it was experimentally confirmed that the vertical component of the magnetic field $\left(H \cos \vartheta_{H}\right)$ is the critical parameter to inhibit the onset of convection as it is included in the Chandrasekhar number Q. According to the experimental and theoretical evidence, it is well known that rotation and magnetic field have a stabilizing effect on the Rayleigh-Bénard problem when they act separately $[2,4,5,7]$.

When rotation and magnetic field act simultaneously and when both of them are parallel to the gravity vector, the stabilizing effects have conflicting tendencies; that is, the results reveal some very unexpected features showing the complex behaviour of the flow; thus, it has been found that the total effect is not always stabilizing with respect to both fields $[1,4,8,9]$. The conflictual behaviour depends on the values of the parameters of the system: rotation rate (Taylor number $T$ ) and magnetic parameter (Chandrasekhar number $Q$ ). Reference [4] only shows explicit results on the dependence of the critical Rayleigh number $R$ on $Q$ and $T$, for the problem in which the conducting liquid is located between two free boundaries, and when the medium adjoining the fluid is nonconducting, because in this situation the system of equations can be solved explicitly.

To the knowledge of the authors, the solution of the problem, in which the three implicated vectors $(\mathbf{g}, \mathbf{H}$, and $\mathbf{\Omega})$ are not parallel, has not been previously reported. In this paper we show, for the rotating-magnetic Rayleigh-Bénard problem with rigid and free surfaces and nonconducting boundaries, the influence on the critical Rayleigh number $R$ of the cooperative work (or competition), between the strength of the magnetic field and the Coriolis force, when they act simultaneously, but in different directions relatively with the gravity vector. In the literature, very few investigations have been carried out aimed to study the cooperative work of perturbations in the flow to inhibit the thermal convection of an infinite layer of fluid; for example, $[7,10,11]$ have found that when the layer of fluid is simultaneously subject to rotation and to a salt concentration field, the stabilizing effects are cumulative; that is, rotation and salt concentration (salted from below) show a cooperative behaviour.

By using the Tau Chebyshev method, we find that depending on the value of the parameters $C_{R}=a_{x} \tan \vartheta_{R}$ and $C_{H}=a_{x} \tan \vartheta_{H}$, a critical situation may appear, at which the competition between $\mathbf{H}$ and $\boldsymbol{\Omega}$ conduct to an unstable system; however as the values of $C_{R}$ or $C_{H}$ are modified, a cooperative work between $\mathbf{H}$ and $\boldsymbol{\Omega}$ may appear which leads to a system in which the thermal convection of the layer of fluid is inhibited.

The paper is organized as follows. In Section 2 we briefly describe the physical model of the problem under study. In Section 3 we formulate the general perturbation equations, from which we get the solution of the problems 1 to 6 described in Table 1. Note that the classical linear problems 1 to 5 of this table have been previously solved by [1], while the linear problem 6 of Table 1 is the subject of the present paper that is, thermal instability analysis of a fluid layer subject to gravity $\mathbf{g}$, rotation $\boldsymbol{\Omega}$, and magnetic field $\boldsymbol{H}$ vectors, with angles $\vartheta_{R}$ and $\vartheta_{H}$ different from zero. In Section 4 we describe the Tau Chebyshev spectral method that is used to solve the set of perturbation equations. Section 5 shows the solution of the six formulated linear problems by using the Tau Chebyshev method, together with a comparison of our calculations with the solution of the classical problems previously published in the literature; see problems 1 to 5 of Table 1. Finally, in Section 6 we present our concluding remarks.

\section{Physical Situation}

Figure 1 shows a schematic representation of the geometry under study. A layer of fluid of constant thickness $\widehat{D}=$ $2 \widehat{d}$ parallel to the $x_{1} x_{2}$-plane with no lateral boundaries is considered. The upper and lower boundaries are located at 


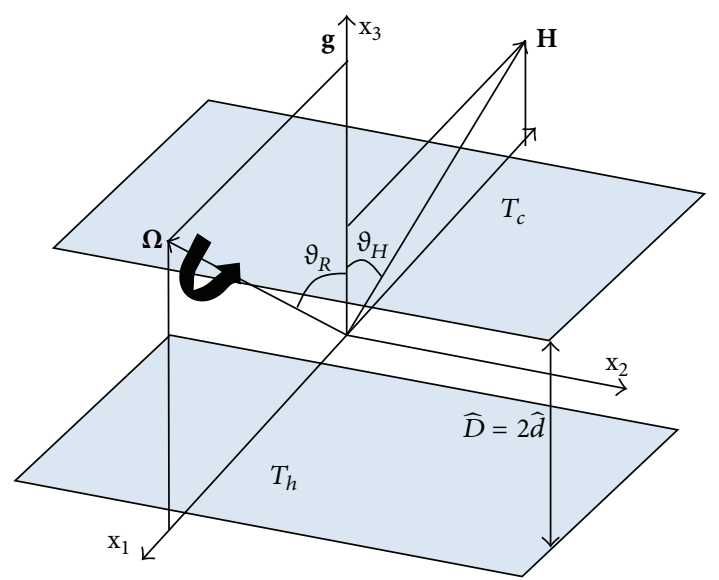

FIGURE 1: Thermal instability analysis in the presence of a rotation vector $\boldsymbol{\Omega}$ and a magnetic field $\mathbf{H}$. The gravity vector $\mathbf{g}$ acts in the negative $x_{3}$-direction. The temperatures $T_{h}$ and $T_{c}$ (where $T_{h}>T_{c}$ ) are applied at the lower and upper boundaries, respectively. The distance between the two surfaces is $\widehat{D}=2 \widehat{d}$. The angle between $\mathbf{g}$ and $\boldsymbol{\Omega}$ is $\vartheta_{R}$, while the angle between $\mathbf{g}$ and $\mathbf{H}$ is $\vartheta_{H}$. Note that the vectors $\Omega$ and $\mathbf{H}$ lay in the $x_{1} x_{3}$-plane. The surfaces are electrically nonconducting; thus, no currents can cross the boundary.

$x_{3}= \pm \hat{d}$. The temperatures $T_{h}$ and $T_{c}$ (where $T_{h}>T_{c}$ ) are applied at the lower and upper boundaries, respectively. The whole layer can rotate with an angular velocity vector $\boldsymbol{\Omega}$, which can be inclined at an angle $\vartheta_{R}$ to the direction of the vertical $x_{3}$-axis, in such a way that $\Omega$ lies in the $x_{1} x_{3}$ plane. Gravity acts in the negative $x_{3}$-direction $\mathbf{g}=-g \mathbf{i}_{3}$. The fluid layer is assumed to be located in a magnetic field represented by the vector $\mathbf{H}$, which can also be inclined at an angle $\vartheta_{H}$ to the direction of the vertical $x_{3}$-axis, in such a way that $\mathbf{H}$ also lies in the $x_{1} x_{3}$-plane. The surfaces are electrically nonconducting; thus, no currents can cross the boundary, and hence we must require $J_{3}=0$, where $J_{3}$ is the third component of the current density vector $\mathbf{J}$.

\section{Thermal Instability Analysis in the Presence of a Rotation Vector and a Magnetic Field}

In this section we analyse the linear thermal instability of a rotating infinite layer of an incompressible, electrically conducting fluid upon which a uniform magnetic field is impressed. The gravity vector $\mathbf{g}$, the rotation vector $\boldsymbol{\Omega}$, and the magnetic field vector $\mathbf{H}$ may act in different directions.

3.1. Governing Equations. Let us consider an infinite fluid layer of an electrically conducting fluid upon which a uniform magnetic field $\mathbf{H}$ is impressed. The fluid is also subjected to rotation about a fixed axis. For an incompressible conducting fluid, the linear convective thermal stability of a fluid layer (initially at rest and with a steady adverse temperature gradient) is analyzed by solving the following perturbation equations in the Boussinesq approximation and in a rotating frame of [1, page 199]:

$$
\begin{gathered}
\frac{\partial u_{i}}{\partial t}=-\frac{\partial}{\partial x_{i}}(\delta \omega)+\beta \theta g \lambda_{i}+\nu \nabla^{2} u_{i}+2 \epsilon_{i j k} u_{j} \Omega_{k} \\
+\frac{\mu}{4 \pi \rho_{o}} H_{j} \frac{\partial h_{i}}{\partial x_{j}} \\
\frac{\partial \theta}{\partial t}=\widehat{\beta} \lambda_{j} u_{j}+\alpha \nabla^{2} \theta, \\
\frac{\partial u_{i}}{\partial x_{i}}=0 \\
\frac{\partial h_{i}}{\partial t}=H_{j} \frac{\partial u_{i}}{\partial x_{j}}+\eta \nabla^{2} h_{i}, \\
\frac{\partial h_{i}}{\partial x_{i}}=0,
\end{gathered}
$$

where $t$ is the time, $u_{i}$ are the components of the velocity vector, $v$ denotes the kinematic viscosity, $\Omega_{k}$ are the components of the rotation vector, $\beta$ is the coefficient of volume expansion, $\alpha$ is the thermal diffusivity, $\theta$ is the perturbation in the temperature, $g$ is the acceleration of gravity, $\mu$ is the magnetic permeability, $\eta$ denotes the resistivity, $\mathbf{H}$ is the impressed magnetic field, $\mathbf{h}$ is the perturbation of the magnetic field, $\lambda=(0,0,1)$ is the unit vector in the direction of the vertical, and $\widehat{\beta}$ is the imposed adverse temperature gradient defined as

$$
\widehat{\beta}=\frac{T_{h}-T_{c}}{\Delta x_{3}},
$$

where $T_{h}$ and $T_{c}$ are the temperature of the lower hot surface and the temperature of the upper cold surface, respectively. The last two terms on the right hand side of (1) are the Coriolis force and the Lorentz force, respectively, and the term $\delta \omega$ is defined as

$$
\delta \omega=\frac{\delta p}{\rho_{o}}-\frac{1}{2}|\boldsymbol{\Omega} \times \mathbf{r}|^{2}+\mu \frac{\mathbf{H} \cdot \mathbf{h}}{4 \pi \rho_{o}},
$$

where

$$
\frac{1}{2}|\boldsymbol{\Omega} \times \mathbf{r}|^{2}
$$

is the centrifugal force, $\delta p$ is the perturbation in the pressure, and $\rho_{o}$ is the density at a mean temperature $T_{o}$.

Taking the curl of (1) we may write

$$
\frac{\partial \omega_{i}}{\partial t}=g \beta \epsilon_{i j k} \frac{\partial \theta}{\partial x_{j}} \lambda_{k}+\nu \nabla^{2} \omega_{i}+2 \Omega_{j} \frac{\partial u_{i}}{\partial x_{j}}+\frac{\mu}{4 \pi \rho_{o}} H_{j} \frac{\partial v_{i}}{\partial x_{j}},
$$

where $\omega_{i}$ are the components of the vorticity vector and the vector $\mathbf{v}=v_{i} \mathbf{i}_{i}=\nabla \times \mathbf{h}$ is the current density induced by the perturbation. Taking the curl of (9) once again, we obtain

$$
\begin{aligned}
\frac{\partial \nabla^{2} u_{i}}{\partial t}= & g \beta\left(\lambda_{i} \nabla^{2} \theta-\lambda_{j} \frac{\partial^{2} \theta}{\partial x_{j} \partial x_{i}}\right)+\nu \nabla^{4} u_{i}-2 \Omega_{j} \frac{\partial \omega_{i}}{\partial x_{j}} \\
& +\frac{\mu}{4 \pi \rho_{o}} H_{j} \frac{\partial}{\partial x_{j}} \nabla^{2} h_{i} .
\end{aligned}
$$


Taking the curl of (4) we have

$$
\frac{\partial v_{i}}{\partial t}=H_{j} \frac{\partial \omega_{i}}{\partial x_{j}}+\eta \nabla^{2} v_{i}
$$

Multiplying (2), (4), (9), (10), and (11) by the components $\lambda_{i}$ we get

$$
\begin{gathered}
\frac{\partial \theta}{\partial t}=\widehat{\beta} u_{3}+\alpha \nabla^{2} \theta, \\
\frac{\partial h_{3}}{\partial t}=H_{j} \frac{\partial u_{3}}{\partial x_{j}}+\eta \nabla^{2} h_{3}, \\
\frac{\partial v_{3}}{\partial t}=H_{j} \frac{\partial \omega_{3}}{\partial x_{j}}+\eta \nabla^{2} v_{3}, \\
\frac{\partial \omega_{3}}{\partial t}=\nu \nabla^{2} \omega_{3}+2 \Omega_{j} \frac{\partial u_{3}}{\partial x_{j}}+\frac{\mu}{4 \pi \rho_{o}} H_{j} \frac{\partial v_{3}}{\partial x_{j}}, \\
\frac{\partial t}{u_{3}}=g\left(\frac{\partial^{2} \theta}{\partial x_{1}^{2}}+\frac{\partial^{2} \theta}{\partial x_{2}^{2}}\right)+\nu \nabla^{4} u_{3}-2 \Omega_{j} \frac{\partial \omega_{3}}{\partial x_{j}} \\
+\frac{\mu}{4 \pi \rho_{o}} H_{j} \frac{\partial}{\partial x_{j}} \nabla^{2} h_{3},
\end{gathered}
$$

where $h_{3}, v_{3}, \omega_{3}$, and $u_{3}$ are the $x_{3}$ components of the perturbation of the magnetic field, the current density, the vorticity, and the velocity, respectively. In this investigation the set of equations to be solved are (12)-(16). Let the rotation vector $\Omega$ be inclined at an angle $\vartheta_{R}$ to the direction of the vertical $x_{3}$-axis. Additionally let the magnetic field vector $\mathbf{H}$ be also inclined at an angle $\vartheta_{H}$ to the direction of the vertical $x_{3}$-axis. The $x_{1}$-axis of the Cartesian coordinate system is defined such as the vectors $\boldsymbol{\Omega}$ and $\mathbf{H}$ lie in the $x_{1} x_{3}$-plane; see Figure 1. Thus the unit vector in the direction of the vertical, the rotation vector, and the magnetic field vector are written as

$$
\begin{gathered}
\lambda=(0,0,1), \quad \boldsymbol{\Omega}=\Omega\left(\sin \vartheta_{R}, 0, \cos \vartheta_{R}\right), \\
\mathbf{H}=H\left(\sin \vartheta_{H}, 0, \cos \vartheta_{H}\right),
\end{gathered}
$$

where $\Omega$ and $H$ are the magnitude of the rotation vector $\Omega$ and the magnitude of the magnetic field vector $\mathbf{H}$, respectively. Using (17) into (13)-(16) we obtain

$$
\begin{aligned}
& \frac{\partial h_{3}}{\partial t}=H\left(\cos \vartheta_{H} \frac{\partial}{\partial x_{3}}+\sin \vartheta_{H} \frac{\partial}{\partial x_{1}}\right) u_{3}+\eta \nabla^{2} h_{3}, \\
& \frac{\partial v_{3}}{\partial t}=H\left(\cos \vartheta_{H} \frac{\partial}{\partial x_{3}}+\sin \vartheta_{H} \frac{\partial}{\partial x_{1}}\right) \omega_{3}+\eta \nabla^{2} v_{3},
\end{aligned}
$$

$$
\begin{aligned}
\frac{\partial \omega_{3}}{\partial t}= & \nu \nabla^{2} \omega_{3}+\frac{\mu}{4 \pi \rho_{o}} H\left(\cos \vartheta_{H} \frac{\partial}{\partial x_{3}}+\sin \vartheta_{H} \frac{\partial}{\partial x_{1}}\right) v_{3} \\
+ & 2 \Omega\left(\cos \vartheta_{R} \frac{\partial}{\partial x_{3}}+\sin \vartheta_{R} \frac{\partial}{\partial x_{1}}\right) u_{3}, \\
\frac{\partial \nabla^{2} u_{3}}{\partial t}= & g \beta\left(\frac{\partial^{2} \theta}{\partial x_{1}^{2}}+\frac{\partial^{2} \theta}{\partial x_{2}^{2}}\right)+\nu \nabla^{4} u_{3} \\
& +\frac{\mu}{4 \pi \rho_{o}} H\left(\cos \vartheta_{H} \frac{\partial}{\partial x_{3}}+\sin \vartheta_{H} \frac{\partial}{\partial x_{1}}\right) \nabla^{2} h_{3} \\
& -2 \Omega\left(\cos \vartheta_{R} \frac{\partial}{\partial x_{3}}+\sin \vartheta_{R} \frac{\partial}{\partial x_{1}}\right) \omega_{3} .
\end{aligned}
$$

3.1.1. The Analysis into Normal Modes. We analyse the perturbations $\theta, h_{3}, v_{3}, \omega_{3}$, and $u_{3}$ into two dimensional periodic waves characterized by a particular wave number $k$. We assume the forms of the perturbations as

$$
\begin{aligned}
& \theta=\Theta\left(x_{3}\right) \exp \left[i\left(k_{x} x_{1}+k_{y} x_{2}\right)+p t\right], \\
& h_{3}=K\left(x_{3}\right) \exp \left[i\left(k_{x} x_{1}+k_{y} x_{2}\right)+p t\right], \\
& v_{3}=X\left(x_{3}\right) \exp \left[i\left(k_{x} x_{1}+k_{y} x_{2}\right)+p t\right], \\
& \omega_{3}=Z\left(x_{3}\right) \exp \left[i\left(k_{x} x_{1}+k_{y} x_{2}\right)+p t\right], \\
& u_{3}=W\left(x_{3}\right) \exp \left[i\left(k_{x} x_{1}+k_{y} x_{2}\right)+p t\right] .
\end{aligned}
$$

If we define the wave number of the disturbance $k$ as $k=\left(k_{x}^{2}+\right.$ $\left.k_{y}^{2}\right)^{1 / 2}$ and $p$ as a constant, (12) and (18) can be written as

$$
\Theta p=\widehat{\beta} W+\alpha\left[\frac{d^{2}}{d x_{3}^{2}}-k^{2}\right] \Theta,
$$

$$
\begin{gathered}
K p=H \cos \vartheta_{H} \frac{d W}{d x_{3}}+H \sin \vartheta_{H} W k_{x} i+\eta\left[\frac{d^{2}}{d x_{3}^{2}}-k^{2}\right] K, \\
X p=H \cos \vartheta_{H} \frac{d Z}{d x_{3}}+H \sin \vartheta_{H} Z k_{x} i+\eta\left[\frac{d^{2}}{d x_{3}^{2}}-k^{2}\right] X \\
Z p=\nu\left[\frac{d^{2}}{d x_{3}^{2}}-k^{2}\right] Z+\frac{\mu}{4 \pi \rho_{o}} H \cos \vartheta_{H} \frac{d X}{d x_{3}} \\
+\frac{\mu}{4 \pi \rho_{o}} H \sin \vartheta_{H} X k_{x} i+2 \Omega \cos \vartheta_{R} \frac{d W}{d x_{3}} \\
+2 \Omega \sin \vartheta_{R} W k_{x} i,
\end{gathered}
$$




$$
\begin{aligned}
{\left[\frac{d^{2}}{d x_{3}^{2}}-k^{2}\right] W p=} & -g \beta k^{2} \Theta+\nu\left[\frac{d^{2}}{d x_{3}^{2}}-k^{2}\right]^{2} W \\
& +\frac{\mu}{4 \pi \rho_{o}} H \cos \vartheta_{H} \frac{d}{d x_{3}}\left[\frac{d^{2}}{d x_{3}^{2}}-k^{2}\right] K \\
& +\frac{\mu}{4 \pi \rho_{o}} H \sin \vartheta_{H}\left[\frac{d^{2}}{d x_{3}^{2}}-k^{2}\right] K k_{x} i \\
& -2 \Omega \cos \vartheta_{R} \frac{d Z}{d x_{3}}-2 \Omega \sin \vartheta_{R} Z k_{x} i .
\end{aligned}
$$

As it is shown in Figure 1, the origin of the coordinate system is located at the middle between the two surfaces; hence, the distance between the two surfaces is $\widehat{D}=2 \widehat{d}$. If we define the following nondimensional variables

$$
\begin{gathered}
a=k \widehat{D}, \quad \sigma=\frac{p \widehat{D}^{2}}{v}, \quad x_{3}^{*}=\frac{2 x_{3}}{\widehat{D}}, \\
\operatorname{Pr}=\frac{v}{\alpha}, \quad \operatorname{Pr}_{2}=\frac{\nu}{\eta},
\end{gathered}
$$

(20) is written as

$$
\begin{aligned}
& {\left[4 D^{2}-a^{2}-\sigma \operatorname{Pr}\right] \Theta=-\left(\frac{\widehat{D}^{2} \widehat{\beta}}{\alpha}\right) W,} \\
& {\left[4 D^{2}-a^{2}-\sigma \operatorname{Pr}_{2}\right] K=-\frac{2 \widehat{D}}{\eta} H \cos \vartheta_{H} D W} \\
& -\frac{\widehat{D}}{\eta} H \sin \vartheta_{H} a_{x} i W \\
& {\left[4 D^{2}-a^{2}-\sigma \operatorname{Pr}_{2}\right] X=-\frac{2 \widehat{D}}{\eta} H \cos \vartheta_{H} D Z} \\
& -\frac{\widehat{D}}{\eta} H \sin \vartheta_{H} a_{x} i Z \\
& {\left[4 D^{2}-a^{2}-\sigma\right] Z=-\frac{4 \widehat{D}}{\nu} \Omega \cos \vartheta_{R} D W-\frac{2 \widehat{D}}{\nu} \Omega \sin \vartheta_{R} a_{x} i W} \\
& -\frac{\mu \widehat{D}}{2 \pi \rho_{o} \nu} H \cos \vartheta_{H} D X \\
& -\frac{\mu \widehat{D}}{4 \pi \rho_{o} \nu} H \sin \vartheta_{H} a_{x} i X,
\end{aligned}
$$

$$
\begin{aligned}
& {\left[4 D^{2}-a^{2}\right]\left[4 D^{2}-a^{2}-\sigma\right] W} \\
& +\frac{\mu \widehat{D}}{2 \pi \rho_{o} \nu} H \cos \vartheta_{H} D\left[4 D^{2}-a^{2}\right] K \\
& +\frac{\mu \widehat{D}}{4 \pi \rho_{o} \nu} H \sin \vartheta_{H}\left[4 D^{2}-a^{2}\right] a_{x} i K \\
& -\frac{4 \widehat{D}^{3}}{\nu} \Omega \cos \vartheta_{R} D Z-\frac{2 \widehat{D}^{3}}{\nu} \Omega \sin \vartheta_{R} a_{x} i Z \\
& =\frac{\widehat{D}^{2}}{\nu} g \beta a^{2} \Theta,
\end{aligned}
$$

where $a_{x}=\widehat{D} k_{x}$. If instability sets in as stationary convection, $\sigma=0$, we can operate (25) by $\left[4 D^{2}-a^{2}\right]$ and in the resulting equation we use (24) to get (let us assume that $\widehat{D}=2$ )

$$
\begin{aligned}
& {\left[\left(4 D^{2}-a^{2}\right)^{2}-\widehat{Q} D^{2}-\widehat{Q} C_{H} i D+\frac{1}{4} \widehat{Q} C_{H}^{2}\right] Z} \\
& \quad=-T^{1 / 2} D\left[4 D^{2}-a^{2}\right] W-\frac{T^{1 / 2}}{2} C_{R} i\left[4 D^{2}-a^{2}\right] W,
\end{aligned}
$$

where

$$
T=\frac{4 \Omega^{2} \widehat{D}^{4} \cos ^{2} \vartheta_{R}}{v^{2}}
$$

is the Taylor number,

$$
C_{H}=a_{x} \tan \vartheta_{H}, \quad C_{R}=a_{x} \tan \vartheta_{R},
$$

$$
\begin{gathered}
\frac{4 \widehat{D}}{\nu} \Omega \cos \vartheta_{R}=\frac{2}{\widehat{D}} T^{1 / 2}, \quad \frac{2 \widehat{D}}{\nu} \Omega \cos \vartheta_{R}=\frac{T^{1 / 2}}{\widehat{D}}, \\
\widehat{Q}=\frac{\mu \widehat{D}^{2}\left(H \cos \vartheta_{H}\right)^{2}}{\pi \rho_{o} \nu \eta} .
\end{gathered}
$$

If we define the resistivity $\eta$ as

$$
\eta=\frac{1}{4 \pi \mu \widehat{\sigma}}
$$

it is possible to write (31) as

$$
\widehat{Q}=4\left(\frac{\mu^{2}\left(H \cos \vartheta_{H}\right)^{2} \widehat{D}^{2} \widehat{\sigma}}{\rho_{o} \nu}\right)=4 Q,
$$

where the variable $Q$ defined by Chandrasekhar [1] is written as

$$
Q=\frac{\mu^{2}\left(H \cos \vartheta_{H}\right)^{2} \widehat{D}^{2} \widehat{\sigma}}{\rho_{o} \nu} .
$$

We can eliminate $K$ from (26) by using (23), then we obtain

$$
\begin{gathered}
{\left[\left(4 D^{2}-a^{2}\right)^{2}-\widehat{Q} D^{2}-\widehat{Q} C_{H} i D+\frac{1}{4} \widehat{Q} C_{H}^{2}\right] W} \\
-\frac{4 \widehat{D}^{3}}{v} \Omega \cos \vartheta_{R} D Z-\frac{2 \widehat{D}^{3}}{v} \Omega \cos \vartheta_{R} C_{R} i Z \\
=\left(\frac{\widehat{D}^{2} g \beta}{v}\right) a^{2} \Theta .
\end{gathered}
$$


We operate (35) by $\left(4 D^{2}-a^{2}\right)$, and in the resulting equation we use (22); hence, we obtain

$$
\begin{gathered}
\left(4 D^{2}-a^{2}\right)\left\{\left[\left(4 D^{2}-a^{2}\right)^{2}-\widehat{Q} D^{2}-\widehat{Q} C_{H} i D+\frac{1}{4} \widehat{Q} C_{H}^{2}\right] W\right. \\
\left.\quad-\frac{4 \widehat{D}^{3}}{v} \Omega \cos \vartheta_{R} D Z-\frac{2 \widehat{D}^{3}}{v} \Omega \cos \vartheta_{R} C_{R} i Z\right\} \\
=-R a^{2} W,
\end{gathered}
$$

where the Rayleigh number $R$ is defined as

$$
R=\frac{\widehat{D}^{4} g \beta \widehat{\beta}}{\nu \alpha}
$$

From (36) we can define the variable $F$ as

$$
\begin{aligned}
F= & {\left[\left(4 D^{2}-a^{2}\right)^{2}-\widehat{Q} D^{2}-\widehat{Q} C_{H} i D+\frac{1}{4} \widehat{Q} C_{H}^{2}\right] W } \\
& -\frac{4 \widehat{D}^{3}}{v} \Omega \cos \vartheta_{R} D Z-\frac{2 \widehat{D}^{3}}{v} \Omega \cos \vartheta_{R} C_{R} i Z ;
\end{aligned}
$$

hence, (36) is written as

$$
\left(4 D^{2}-a^{2}\right) F=-R a^{2} W .
$$

By using the following relationships

$$
\begin{aligned}
& \frac{4 \widehat{D}^{3}}{v} \Omega \cos \vartheta_{R}=2 \widehat{D} T^{1 / 2}, \\
& \frac{2 \widehat{D}^{3}}{\nu} \Omega \cos \vartheta_{R}=\widehat{D} T^{1 / 2},
\end{aligned}
$$

into (38) we can formulate the relevant equations to be solved as

$$
\begin{gathered}
16 D^{4} W-\left(8 a^{2}+4 Q\right) D^{2} W-4 Q C_{H} i D W+\left(a^{4}+Q C_{H}^{2}\right) W \\
-4 T^{1 / 2} D Z-2 T^{1 / 2} C_{R} i Z-F=0, \\
4 D^{2} F-a^{2} F=-R a^{2} W, \\
16 D^{4} Z-\left(8 a^{2}+4 Q\right) D^{2} Z-4 Q C_{H} i D Z+\left(a^{4}+Q C_{H}^{2}\right) Z \\
+4 T^{1 / 2} D^{3} W-T^{1 / 2} a^{2} D W+2 T^{1 / 2} C_{R} i D^{2} W \\
-\frac{1}{2} T^{1 / 2} C_{R} i a^{2} W=0 .
\end{gathered}
$$

Notice that when the magnetic field is negligible $\mathbf{H}=0$, that is, pure rotation, and the relevant equations to be solved are the following:

$$
\begin{gathered}
16 D^{4} W-8 a^{2} D^{2} W+a^{4} W-4 T^{1 / 2} D Z-2 T^{1 / 2} C_{R} i Z-F=0, \\
4 D^{2} F-a^{2} F=-R a^{2} W \\
16 D^{4} Z-8 a^{2} D^{2} Z+a^{4} Z+4 T^{1 / 2} D^{3} W-T^{1 / 2} a^{2} D W \\
+2 T^{1 / 2} C_{R} i D^{2} W-\frac{1}{2} T^{1 / 2} C_{R} i a^{2} W=0
\end{gathered}
$$

together with the following boundary conditions.

(a) For rigid surfaces at $x_{3}^{*}=-1$ and at $x_{3}^{*}=1$ :

$$
\begin{array}{r}
F=0, \quad Z=0, \quad W=0, \quad D W=0, \\
\text { at } x_{3}^{*}=-1, \text { at } x_{3}^{*}=1 .
\end{array}
$$

(b) For free surfaces at $x_{3}^{*}=-1$ and at $x_{3}^{*}=1$ :

$$
\begin{aligned}
F=0, \quad D Z=0, \quad W & =0, \quad D^{2} W=0, \\
\text { at } x_{3}^{*} & =-1, \text { at } x_{3}^{*}=1 .
\end{aligned}
$$

(It must be pointed out that due to the process we follow to derive the general relevant equations, the vorticity equation (46) has both different form and higher order than the one we obtained by following Chandrasekhar's procedure; see Chandrasekhar [1], Chapter III, page 90. The equation for the vorticity that we obtained by considering only rotation without magnetic field is $4 D^{2} Z-a^{2} Z+T^{1 / 2} D W+(1 / 2) T^{1 / 2} C_{R} i W=$ 0 , which is the same as the one obtained by Chandrasekhar [1].)

On the other hand when the rotation vector $\Omega$ is equal to zero, the relevant equations are

$$
\begin{gathered}
16 D^{4} W-\left(8 a^{2}+4 Q\right) D^{2} W-4 Q i C_{H} D W+\left(a^{4}+Q C_{H}^{2}\right) W \\
-F=0, \\
4 D^{2} F-a^{2} F=-R a^{2} W
\end{gathered}
$$

together with the following boundary conditions.

(a) For rigid surfaces at $x_{3}^{*}=-1$ and at $x_{3}^{*}=1$ :

$F=0, \quad W=0, \quad D W=0, \quad$ at $x_{3}^{*}=-1$, at $x_{3}^{*}=1$.

(b) For free surfaces at $x_{3}^{*}=-1$ and at $x_{3}^{*}=1$ :

$F=0, \quad W=0, \quad D^{2} W=0, \quad$ at $x_{3}^{*}=-1$, at $x_{3}^{*}=1$.

(For this particular case in which $\Omega=0$, the $x_{3}$ component of vorticity $Z$ vanishes, see Chandrasekhar [1], Chapter IV, page 166 ; hence the general equation (43) is not taken into account in the analysis.)

Therefore the boundary conditions needed to solve the general equations (41)-(43) are defined by (47) and (48). 
TABLE 2: Critical Rayleigh numbers and wave numbers at marginal stability for the onset of stationary convection when both bounding surfaces are rigid. Gravity vector $\mathbf{g}$ parallel to the rotation vector $\boldsymbol{\Omega}$. $T$ is the Taylor number. $R$ is the Rayleigh number obtained by the Tau Chebyshev method. $R_{\mathrm{Ch}}$ is the Rayleigh number obtained by Chandrasekhar [1].

\begin{tabular}{lccc}
\hline$T$ & $a$ & $R$ & $R_{\mathrm{Ch}}$ \\
\hline 10 & 3.1 & $1.7127 E 3$ & $1.713 E 3$ \\
$10^{2}$ & 3.15 & $1.756 E 3$ & $1.7566 E 3$ \\
$10^{3}$ & 3.50 & $2.1514 E 3$ & $2.1517 E 3$ \\
$10^{5}$ & 7.20 & $1.6720 E 4$ & $1.6721 E 4$ \\
$10^{10}$ & 55.5 & $4.476 E 7$ & $3.4574 E 7$ \\
\hline
\end{tabular}

\section{The Tau Chebyshev Numerical Method}

In order to find an approximate solution to the set of perturbations equations formulated in the previous section, together with the boundary conditions, we use expansions in Chebyshev polynomials. Therefore we expand the variables $W\left(x_{3}^{*}\right), F\left(x_{3}^{*}\right)$, and $Z\left(x_{3}^{*}\right)$ as well as their derivatives $D=$ $d / d x_{3}^{*}, D^{2}=d^{2} / d x_{3}^{* 2}, D^{3}=d^{3} / d x_{3}^{* 3}$, and $D^{4}=d^{4} / d x_{3}^{* 4}$, in the interval $-1 \leq x_{3}^{*} \leq 1$ as follows:

$$
\begin{gathered}
W\left(x_{3}^{*}\right)=\sum_{n=0}^{N} a_{W n} T_{n}\left(x_{3}^{*}\right), \\
F\left(x_{3}^{*}\right)=\sum_{n=0}^{N} a_{F n} T_{n}\left(x_{3}^{*}\right), \\
Z\left(x_{3}^{*}\right)=\sum_{n=0}^{N} a_{Z n} T_{n}\left(x_{3}^{*}\right), \\
\frac{d^{q} W}{d x_{3}^{* q}}=\sum_{n=0}^{N} a_{W n} \frac{d^{q} T_{n}\left(x_{3}^{*}\right)}{d x_{3}^{* q}}=\sum_{n=0}^{N} b_{W n} T_{n}\left(x_{3}^{*}\right), \\
\frac{d^{q} F}{d x_{3}^{* q}}=\sum_{n=0}^{N} a_{F n} \frac{d^{q} T_{n}\left(x_{3}^{*}\right)}{d x_{3}^{* q}}=\sum_{n=0}^{N} b_{F n} T_{n}\left(x_{3}^{*}\right), \\
\frac{d^{q} Z}{d x_{3}^{* q}}=\sum_{n=0}^{N} a_{Z n} \frac{d^{q} T_{n}\left(x_{3}^{*}\right)}{d x_{3}^{* q}}=\sum_{n=0}^{N} b_{Z n} T_{n}\left(x_{3}^{*}\right),
\end{gathered}
$$

where $q$ is an integer number defining the order of the derivative of the functions $W, F$, and $Z$, and $T_{n}\left(x_{3}^{*}\right)$ are the $n$ th-degree Chebyshev polynomials of the first kind (with $N$ as the maximum degree used for the polynomials). The Chebyshev polynomials satisfy the orthogonality relation [12-14]:

$$
\int_{-1}^{1} T_{n}\left(x_{3}^{*}\right) T_{m}\left(x_{3}^{*}\right)\left(1-x_{3}^{* 2}\right)^{-1 / 2} d x_{3}^{*}=\frac{\pi}{2} c_{n} \delta_{n m}
$$

where $c_{0}=2, c_{n}=1$ for $n>0$. Equations for the expansion coefficients $a_{W n}, a_{F n}, a_{Z n}, b_{W n}$ (in terms of $a_{W n}$ ), $b_{F n}$ (in terms of $a_{F n}$ ), and $b_{Z n}$ (in terms of $a_{Z n}$ ) are generated by substituting (52)-(57) into the set of perturbation equations shown in the previous section and applying the orthogonality property
(58). In the literature it is possible to find explicit formulae for the expansion coefficients $b_{W n}$ in terms of $a_{W n}, b_{F n}$ in terms of $a_{F n}$, and $b_{Z n}$ in terms of $a_{Z n}[12,15]$.

The method that we have selected to obtain the equations for the coefficients $a_{W n}, a_{F n}$, and $a_{Z n}$ is the Tau Chebyshev method. The Tau Chebyshev method is an efficient numerical procedure for finding eigenvalues and eigenfunctions of systems in fluid and solid mechanics theories. One advantage of the algorithm is that it is able to calculate as many eigenvalues as required, not just the dominant one $[13,14]$. Using this numerical methodology we generate, by substituting (52), (53), (55), and (56) into the couple of the general perturbation equations for the case when $\Omega=0$; see (49), one equation for each $n=0,1,2, \ldots, 2 N+2-6$. The remaining six equations, those for $2 N+2-6+1 \leq n \leq 2 N+2$, are generated by using the six boundary conditions. Hence for rigid surfaces, the set of boundary conditions are written as

$$
\begin{gathered}
W(1)=\sum_{n=0}^{N} a_{W n}=0, \quad \frac{d W(1)}{d x_{3}^{*}}=\sum_{n=0}^{N} a_{W n} n^{2}=0, \\
W(-1)=\sum_{n=0}^{N} a_{W n}(-1)^{n}=0, \\
\frac{d W(-1)}{d x_{3}^{*}}=\sum_{n=0}^{N} a_{W n}(-1)^{n-1} n^{2}=0, \\
F(1)=\sum_{n=0}^{N} a_{F n}=0, \quad F(-1)=\sum_{n=0}^{N} a_{F n}(-1)^{n}=0,
\end{gathered}
$$

whereas for free surfaces the set of boundary conditions are written as

$$
\begin{gathered}
W(1)=\sum_{n=0}^{N} a_{W n}=0, \\
\frac{d^{2} W(1)}{d x_{3}^{* 2}}=\sum_{n=0}^{N} a_{W n} n^{2}\left(\frac{n^{2}-1}{3}\right)=0, \\
W(-1)=\sum_{n=0}^{N} a_{W n}(-1)^{n}=0, \\
\frac{d^{2} W(-1)}{d x_{3}^{* 2}}=\sum_{n=0}^{N} a_{W n}(-1)^{n+2} n^{2}\left(\frac{n^{2}-1}{3}\right)=0, \\
F(1)=\sum_{n=0}^{N} a_{F n}=0, \quad F(-1)=\sum_{n=0}^{N} a_{F n}(-1)^{n}=0 .
\end{gathered}
$$

Using the Tau Chebyshev method in the cases with rotation $(\Omega \neq 0)$ we generate, by substituting (52)-(57) into the three perturbation equations, one equation for each $n=$ $0,1,2, \ldots, 3 N+3-8$. The remaining eight equations, those for $3 N+3-8+1 \leq n \leq 3 N+3$, are generated by using 
TABLE 3: Critical Rayleigh numbers and wave numbers at marginal stability for the onset of stationary convection when both bounding surfaces are free. Gravity vector $\mathbf{g}$ parallel to the rotation vector $\boldsymbol{\Omega}$. T is the Taylor number. $R$ is the Rayleigh number obtained by the Tau Chebyshev method. $R_{\mathrm{Ch}}$ is the Rayleigh number obtained by Chandrasekhar [1].

\begin{tabular}{lcccccc}
\hline$T$ & $a$ & $R$ & $R_{\mathrm{Ch}}$ & $T$ & $a$ & $R$ \\
\hline $10^{2}$ & 2.594 & $8.262 E 2$ & $8.263 E 2$ & $10^{6}$ & 12.86 & $9.222 E 4$ \\
$10^{3}$ & 3.710 & $1.676 E 3$ & $1.676 E 3$ & $10^{8}$ & 28.02 & $1.897 E 6$ \\
$10^{4}$ & 5.698 & $5.377 E 3$ & $5.377 E 3$ & $10^{11}$ & 88.87 & $1.875 E 8$ \\
$10^{5}$ & 8.626 & $2.130 E 4$ & $2.131 E 4$ & $10^{13}$ & 191.51 & $4.037 E 9$ \\
\hline
\end{tabular}

TABLE 4: Thermal instability analysis in the presence of a rotation vector when both bounding surfaces are rigid: gravity vector and rotation vector acting in different directions. Rayleigh numbers $R$ for the Taylor number $T=100$ and for various values of $a$ and $C_{R}$.

\begin{tabular}{cccccc}
\hline$C_{R}$ & $a$ & $R$ & $C_{R}$ & $a$ & $R$ \\
\hline 0.0 & 3.15 & 1756.37 & - & - & - \\
\hline \multirow{3}{*}{0.5} & 3.0 & 1765.91 & & 3.0 & 1806.85 \\
& 3.15 & 1758.85 & 2.0 & 3.15 & 1796.01 \\
& 3.35 & 1767.31 & & 3.35 & 1800.20 \\
\hline \multirow{3}{*}{1.0} & 3.0 & 1774.10 & & 3.0 & 1937.84 \\
& 3.15 & 1766.28 & 4.0 & 3.35 & 1905.43 \\
& 3.35 & 1773.89 & & 3.45 & 1908.84 \\
\hline
\end{tabular}

TABLE 5: Thermal instability analysis in the presence of a rotation vector when both bounding surfaces are free: Gravity vector and rotation vector acting in different directions. Rayleigh numbers $R$ for the Taylor number $T=100$ and for various values of $a$ and $C_{R}$.

\begin{tabular}{cccccc}
\hline$C_{R}$ & $a$ & $R$ & $C_{R}$ & $a$ & $R$ \\
\hline 0.0 & 2.594 & 826.28 & - & - & - \\
\hline \multirow{3}{*}{0.5} & 2.5 & 836.54 & & 2.62 & 943.16 \\
& 2.6 & 833.77 & 2.0 & 2.74 & 932.00 \\
& 2.64 & 833.84 & & 3.00 & 933.42 \\
\hline \multirow{4}{*}{1.0} & 2.5 & 861.92 & & 2.76 & 1228.56 \\
& 2.66 & 855.00 & 4.0 & 3.2 & 1145.92 \\
& 2.72 & 855.35 & & 3.4 & 1151.32 \\
\hline
\end{tabular}

TABLE 6: Critical Rayleigh numbers and wave numbers at marginal stability for the onset of stationary convection when both bounding surfaces are rigid. Gravity vector $\mathbf{g}$ parallel to the magnetic field vector $\mathbf{H} . \mathbf{Q}$ is the dimensionless magnetic parameter. $R$ is the Rayleigh number obtained by the Tau Chebyshev method. $R_{\mathrm{Ch}}$ is the Rayleigh number obtained by Chandrasekhar [1].

\begin{tabular}{lccc}
\hline$Q$ & $a$ & $R$ & $R_{\mathrm{Ch}}$ \\
\hline 10 & 3.25 & 1945.8 & 1945.8 \\
100 & 4.00 & 3757.27 & 3757.3 \\
1000 & 5.80 & 17102.94 & 17103 \\
4000 & 7.40 & 54696.93 & 54700 \\
10000 & 8.66 & 124508.47 & 124509 \\
\hline
\end{tabular}

the eight boundary conditions. Hence for rigid surfaces, the set of boundary conditions are written as

$$
\begin{gathered}
W(1)=\sum_{n=0}^{N} a_{W n}=0, \quad \frac{d W(1)}{d x_{3}^{*}}=\sum_{n=0}^{N} a_{W n} n^{2}=0, \\
W(-1)=\sum_{n=0}^{N} a_{W n}(-1)^{n}=0, \\
\frac{d W(-1)}{d x_{3}^{*}}=\sum_{n=0}^{N} a_{W n}(-1)^{n-1} n^{2}=0, \\
F(1)=\sum_{n=0}^{N} a_{F n}=0, \quad F(-1)=\sum_{n=0}^{N} a_{F n}(-1)^{n}=0, \\
Z(1)=\sum_{n=0}^{N} a_{Z n}=0, \quad Z(-1)=\sum_{n=0}^{N} a_{Z n}(-1)^{n}=0,
\end{gathered}
$$

whereas for free surfaces the set of boundary conditions are written as

$$
\begin{aligned}
& W(1)=\sum_{n=0}^{N} a_{W n}=0, \\
& \frac{d^{2} W(1)}{d x_{3}^{* 2}}=\sum_{n=0}^{N} a_{W n} n^{2}\left(\frac{n^{2}-1}{3}\right)=0, \\
& W(-1)=\sum_{n=0}^{N} a_{W n}(-1)^{n}=0, \\
& \frac{d^{2} W(-1)}{d x_{3}^{* 2}}=\sum_{n=0}^{N} a_{W n}(-1)^{n+2} n^{2}\left(\frac{n^{2}-1}{3}\right)=0, \\
& F(1)=\sum_{n=0}^{N} a_{F n}=0, \\
& F(-1)=\sum_{n=0}^{N} a_{F n}(-1)^{n}=0 \\
& \frac{d Z(1)}{d x_{3}^{*}}=\sum_{n=0}^{N} a_{Z n} n^{2}=0, \\
& \frac{d Z(-1)}{d x_{3}^{*}}=\sum_{n=0}^{N} a_{Z n}(-1)^{n-1} n^{2}=0 .
\end{aligned}
$$


TABLE 7: Critical Rayleigh numbers and wave numbers at marginal stability for the onset of stationary convection when both bounding surfaces are free. Gravity vector $\mathbf{g}$ parallel to the magnetic field vector $\mathbf{H}$. $Q$ is the dimensionless magnetic parameter. $R$ is the Rayleigh number obtained by the Tau Chebyshev method. $R_{\mathrm{Ch}}$ is the Rayleigh number obtained by Chandrasekhar [1].

\begin{tabular}{|c|c|c|c|c|c|c|c|}
\hline$Q$ & $a$ & $R$ & $R_{\mathrm{Ch}}$ & Q & $a$ & $R$ & $R_{\mathrm{Ch}}$ \\
\hline 10 & 2.590 & 923.069 & 923.070 & 7500 & 8.159 & 91705.75 & 91705.7 \\
\hline 100 & 3.702 & 2653.708 & 2653.71 & 9000 & 8.429 & 108622.9 & 108623 \\
\hline 1000 & 5.684 & 15207.01 & 15207.0 & 15000 & 9.227 & 175279.33 & 175279 \\
\hline 2000 & 6.453 & 27699.95 & 27699.9 & 17000 & 9.433 & 197248.97 & 197249 \\
\hline 5000 & 7.585 & 63135.98 & 63135.9 & 35000 & 10.70 & 392013.13 & 392013 \\
\hline
\end{tabular}

TABLE 8: Thermal instability analysis in the presence of a magnetic field when both bounding surfaces are rigid: gravity vector and magnetic field vector acting in different directions. Rayleigh numbers for the dimensionless parameter $Q=100$ and for various values of $a$ and $C_{H}$. $R$ is the Rayleigh number obtained by the Tau Chebyshev method. $R_{\mathrm{Ch}}$ is the Rayleigh number obtained by Chandrasekhar [1].

\begin{tabular}{cccccccc}
\hline$C_{H}$ & $a$ & $R$ & $R_{\mathrm{Ch}}$ & $C_{H}$ & $a$ & $R_{\mathrm{Ch}}$ \\
\hline 0.0 & 4.0 & 3757.27 & 3757.3 & - & - & - \\
\hline \multirow{3}{*}{0.5} & 3.9 & 3785.12 & 3797.0 & & 3.9 & 4146.18 & 4190.0 \\
& 4.0 & 3780.70 & 3793.0 & 2.0 & 4.0 & 4136.55 & 4181.0 \\
& 4.1 & 3782.82 & 3795.0 & & 4.1 & 4133.94 & 5356.98 \\
1.0 & 3.9 & 3856.64 & 3875.0 & & 3.9 & 5328.39 \\
& 4.0 & 3851.20 & 3878.0 & 4.0 & 4.0 & 5308.46 \\
\hline
\end{tabular}

TABLE 9: Thermal instability analysis in the presence of a magnetic field when both bounding surfaces are free: gravity vector and magnetic field vector acting in different directions. Rayleigh numbers for the dimensionless parameter $Q=100$, and for various values of $a$ and $C_{H} . R$ is the Rayleigh number obtained by the Tau Chebyshev method. $R_{\mathrm{Ch}}$ is the Rayleigh number obtained by Chandrasekhar [1].

\begin{tabular}{cccccc}
\hline$C_{H}$ & $a$ & $R$ & $C_{H}$ & $a$ & $R$ \\
\hline 0.0 & 3.702 & 2653.70 & - & - & - \\
\hline \multirow{3}{*}{0.5} & 3.6 & 2679.49 & & 3.6 & 3028.72 \\
& 3.7 & 2676.17 & 2.0 & 3.8 & 3017.01 \\
& 3.8 & 2678.55 & & 3.9 & 3020.24 \\
\hline \multirow{4}{*}{1.0} & 3.6 & 2748.31 & & 3.6 & 4226.99 \\
& 3.7 & 2743.9 & 4.0 & 4.1 & 4151.21 \\
& 3.8 & 2745.3 & & 4.2 & 4157.48 \\
\hline
\end{tabular}

Notice that the high frequency behaviour (i.e., high $n$ ) of the solution is not governed by the perturbation equations but by the boundary conditions [12]. The Tau equations can be written as a generalized eigenvalue problem $(\mathbf{A x})=R(\mathbf{B x})$, where the vector $\mathbf{x}$ includes the coefficients $a_{W n}, a_{F n}$ (and $\left.a_{Z n}\right), R$ is the Rayleigh number, and $\mathbf{A}$ and $\mathbf{B}$ are $(2 N+2) \times$ $(2 N+2)$ matrices (or $(3 N+3) \times(3 N+3)$ matrices) whose first $2 N+2-6$ (or $3 N+3-8$ ) rows are defined by the two (or three) perturbation equations. The last six (or eight) rows of $\mathbf{A}$ are given by the boundary conditions, and the last six (or eight) rows of $\mathbf{B}$ vanish [16]. The system of equations has been solved numerically by using the routine F02GJF from the NAG library. The routine F02GJF calculates all the eigenvalues of the eigenproblem $\mathbf{A x}=\hat{\lambda} \mathbf{B x}$, by using the $Q Z$ algorithm (see http://www.nag.co.uk/numeric/Fl/manual/ xhtml/F02/f02gjf.xml).

\section{Results}

5.1. Thermal Instability Analysis in the Presence of a Rotation Vector, $\vartheta_{R}=0, \mathbf{H}=0$. Tables 2 and 3 show the critical Rayleigh number $R$ and wave number $a$ of the unstable modes at marginal stability for the onset of stationary convection when both bounding surfaces are rigid and when both bounding surfaces are free, respectively. It is possible to observe that the results obtained by the Tau Chebyshev method are in full agreement with the variational formulation; see [1]. It is also observed the inhibition effect of the Coriolis force as the Taylor number $T$ increases. As $T$ becomes greater, the value of the critical wave number also increases.

5.2. Thermal Instability Analysis in the Presence of a Rotation Vector, $\vartheta_{R} \neq 0, \mathbf{H}=0$. Tables 4 and 5 show the results obtained by the Tau Chebyshev method when both bounding surfaces are rigid and when both bounding surfaces are free, respectively. It is observed that when the Taylor number is $T=100$, as the value of the $C_{R}$ parameter increases, the critical parameters (Rayleigh number $R_{c}$ and wave number $a_{c}$ ) become greater. Note in Table 4 that when $C_{R}=1, a_{c}=$ 3.15 and $R_{c}=1766.28$; however, when $C_{R}=4, a_{c}=3.35$ and $R_{c}=1905.43$. Also note in Table 5 that when $C_{R}=1$, $a_{c}=2.66$ and $R_{c}=855.0$; however, when $C_{R}=4, a_{c}=3.2$ and $R_{c}=1145.92$. Hence for a fixed Taylor number, the net effect of increasing $C_{R}$ is to stabilize the system.

5.3. Thermal Instability Analysis in the Presence of a Magnetic Field, $\vartheta_{H}=0, \boldsymbol{\Omega}=0$. Tables 6 and 7 show the critical Rayleigh number $R$ and wave number $a$ of the unstable modes at marginal stability for the onset of stationary convection 
TABLE 10: Thermal instability analysis in the presence of a rotation and a magnetic field when both bounding surfaces are free: gravity vector $\mathbf{g}$ parallel to both rotation vector $\boldsymbol{\Omega}$ and magnetic field vector $\mathbf{H}$. Critical Rayleigh numbers and wave numbers at marginal stability for the onset of stationary convection. Taylor number $T_{1}=T / \pi^{4}=10^{5}$ for various values of the dimensionless magnetic parameter $Q_{1}=Q / \pi^{2} . R$ and $a$ are the Rayleigh number and the wave number, respectively, obtained by the Tau Chebyshev method. $R_{\mathrm{Ch}}$ and $a_{\mathrm{Ch}}$ are the Rayleigh number and the wave number, respectively, obtained by Chandrasekhar [1]. The superscripts 1 and 2 mean the two minima of the Rayleigh number.

\begin{tabular}{|c|c|c|c|c|c|c|c|c|}
\hline$Q_{1}$ & $a^{1}$ & $R^{1}$ & $a_{\mathrm{Ch}}^{1}$ & $R_{\mathrm{Ch}}^{1}$ & $a^{2}$ & $R^{2}$ & $a_{\mathrm{Ch}}^{2}$ & $R_{\mathrm{Ch}}^{2}$ \\
\hline 10 & 18.87 & $4.066 E 5$ & 18.9 & $4.067 E 5$ & - & - & - & - \\
\hline 50 & 18.58 & $4.028 E 5$ & 18.6 & $4.028 E 5$ & 3.49 & $7.261 E 5$ & 3.48 & $7.261 E 5$ \\
\hline 100 & 18.2 & $3.976 E 5$ & 18.2 & $3.977 E 5$ & 3.365 & $3.932 E 5$ & 3.37 & $3.933 E 5$ \\
\hline 1000 & - & - & - & - & 5.675 & $1.828 E 5$ & 5.67 & $1.829 E 5$ \\
\hline 10000 & - & - & - & - & 12.29 & $1.081 E 6$ & 12.3 & $1.082 E 6$ \\
\hline 100000 & - & - & - & - & 18.9 & $1.015 E 7$ & 18.9 & $1.015 E 7$ \\
\hline
\end{tabular}

when both bounding surfaces are rigid and when both bounding surfaces are free, respectively. It is possible to observe that the results obtained by the Tau Chebyshev method are in full agreement with the variational formulation; see [1]. It is also observed the inhibition effect of the magnetic field, as the magnetic number $Q$ increases. As $Q$ becomes greater, the value of the critical wave number also increases.

\subsection{Thermal Instability Analysis in the Presence of a Magnetic} Field, $\vartheta_{H} \neq 0, \Omega=0$. Tables 8 and 9 show the results obtained by the Tau Chebyshev method when both bounding surfaces are rigid and when both bounding surfaces are free, respectively. It is observed that when the Chandrasekhar number $Q$ is fixed to $Q=100$, as the value of the $C_{H}$ parameter increases, the critical Rayleigh number becomes greater. In Table 8 we present a comparison between the results obtained by [1] and the results of the Tau Chebyshev method. We observe that if the data obtained by Chandrasekhar are the exact values, the maximum percentage error (\% error), when $C_{H} \neq 0$, of the critical $R$ number calculated by the Tau Chebyshev method occurs when $C_{H}=4.0$ and $a=4.1$, with an error of $3 \%$, while the minimum percentage error occurs when $C_{H}=0.5$ and $a=3.9$, with an error of $0.3 \%$. Thus we may conclude that our calculations are in good agreement with Chandrasekhar's results. Note in Table 8 that when $C_{H}=$ 1 , the critical wave number $a=4$; however, when $C_{H}=4$, the critical wave number $a=4.1$ Also note in Table 9 that when $C_{H}=1$, the critical wave number $a=3.7$; however, when $C_{H}=4$, the critical wave number $a=4.1$. Hence for a fixed Chandrasekhar number $Q$, the net effect of increasing $C_{H}$ is to stabilize the system.

5.5. Thermal Instability Analysis in the Presence of a Rotation Vector and a Magnetic Field, $\vartheta_{R}=0, \vartheta_{H}=0$. Table 10 shows a comparison between the results obtained by the Tau Chebyshev method and those of Chandrasekhar's variational formulation when both bounding surfaces are free. The results have been obtained for several values of the modified magnetic number $Q_{1}=Q / \pi^{2}$ and for a fixed value of the modified Taylor number $T_{1}=T / \pi^{4}=10^{5}$. Notice that the agreement is encouraging. Also note that the two minima of the Rayleigh number are successfully reproduced by the Tau
Chebyshev method. It is interesting to mention that by using the Tau Chebyshev method we found two additional critical Rayleigh numbers that were not reported by Chandrasekhar (see [1], Table XVIII, page 205, for $T_{1}=10^{5}$ ). We found (not shown in Table 10) that when $Q_{1}=30, a=3.93$ and $R=1.125 \times 10^{6}$, and when $Q_{1}=200, a=17.1$ and $R=$ $3.855 \times 10^{5}$. Figure 2 shows that if $T_{1}=10^{5}$, when both bounding surfaces are free (see row (a)), and the magnetic parameter $Q_{1}$ is either $Q_{1}=40$ (curve labelled a), $Q_{1}=$ 100 (curve labelled b), or $Q_{1}=200$ (curve labelled c), see left panel, the two minima of the Rayleigh number curves reported by Chandrasekhar are recovered (see [1], Figure 49, page 208). Row (b), left panel of Figure 2, shows that when the bounding surfaces are rigid, the two minima only slightly appear when $Q_{1}=100$ (curve labelled b) and $Q_{1}=200$ (curve labelled c); however, when $Q_{1}=40$ (curve labelled a), only one minimum value is present. Figure 2 shows on the left panel of both rows the case with pure rotation $T_{1}=10^{5}$, that is, without magnetic field (see the curves with circles). It is pointed out that when the surfaces are free (row (a), left panel), the effect of the magnetic field is to destabilize the system, particularly when $Q_{1}=200$ (curve labelled c). This effect is clearly observed for low values of the modified wave number $x=a^{2} / \pi^{2}$. It is also observed that the system with $Q_{1}=200$ is more unstable than the pure rotation case. However when the surfaces are rigid (see row (b), left panel), it is possible to observe that as the magnetic field becomes greater, it firstly induces a stabilizing effect, with respect to the pure rotation case (see curve labelled a for $Q_{1}=40$ and the curve with circles), but for $Q_{1}=100$ and 200, it initiates a destabilizing effect; however, the pure rotation case (see the curve with circles) is more unstable. On the other hand Figure 2 shows, on the right panel of both rows, the influence of greater magnetic numbers $\left(Q_{1}=400\right.$ (see continuous lines), $Q_{1}=1500$ (see dashed lines), $Q_{1}=2000$ (see dotted lines), and $Q_{1}=4000$ (see dash-dotted lines)). Also shown on the right panel of both rows is the case with pure magnetic field $Q_{1}=4000$, that is, without rotation (see the curves with asterisks). It is pointed out that when the surfaces are free or rigid, when $Q_{1}=400$ (see the continuous lines), the system reaches its critical situation; that is, the minimum value of the Reynolds number is attained. However as $Q_{1}$ increases even more, the system becomes more stable. Now when the surfaces are free (row (a)), and when the curve with asterisks 

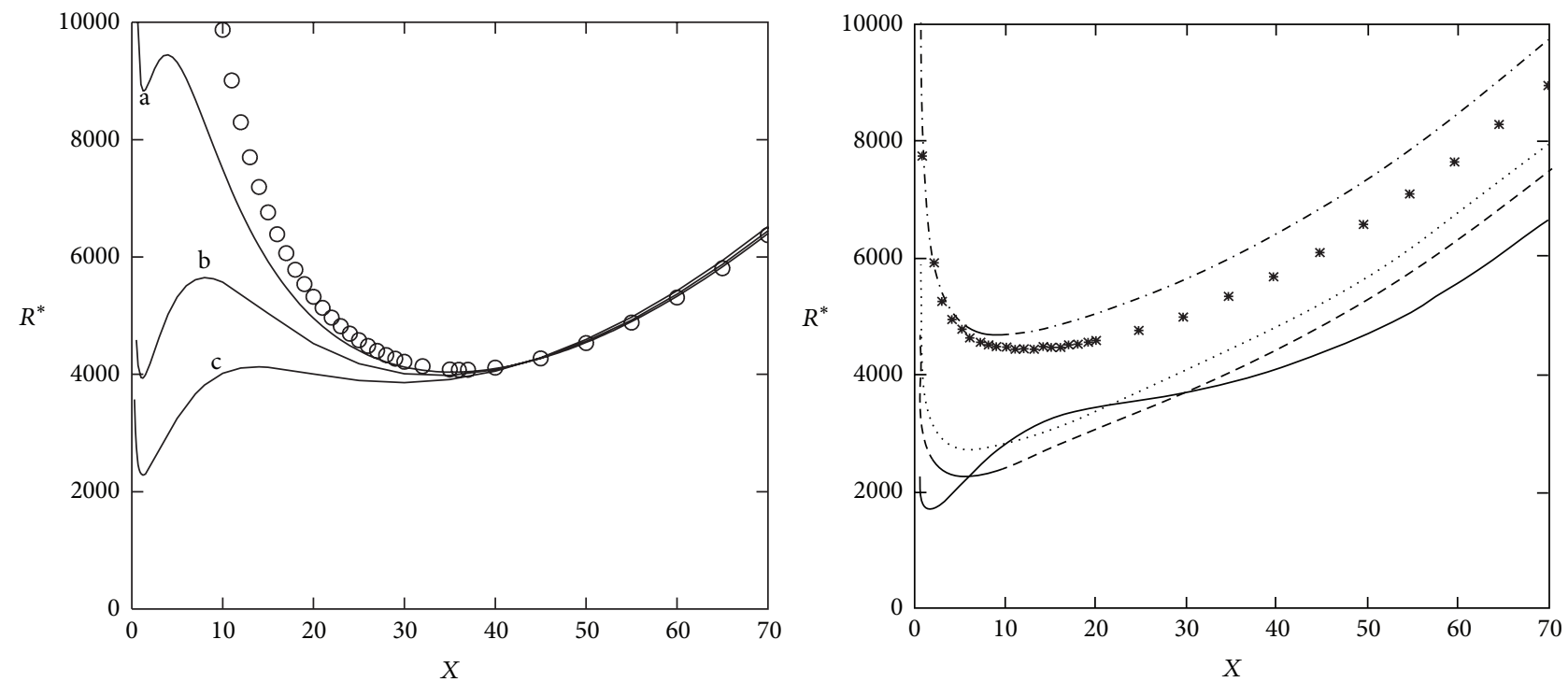

(a)
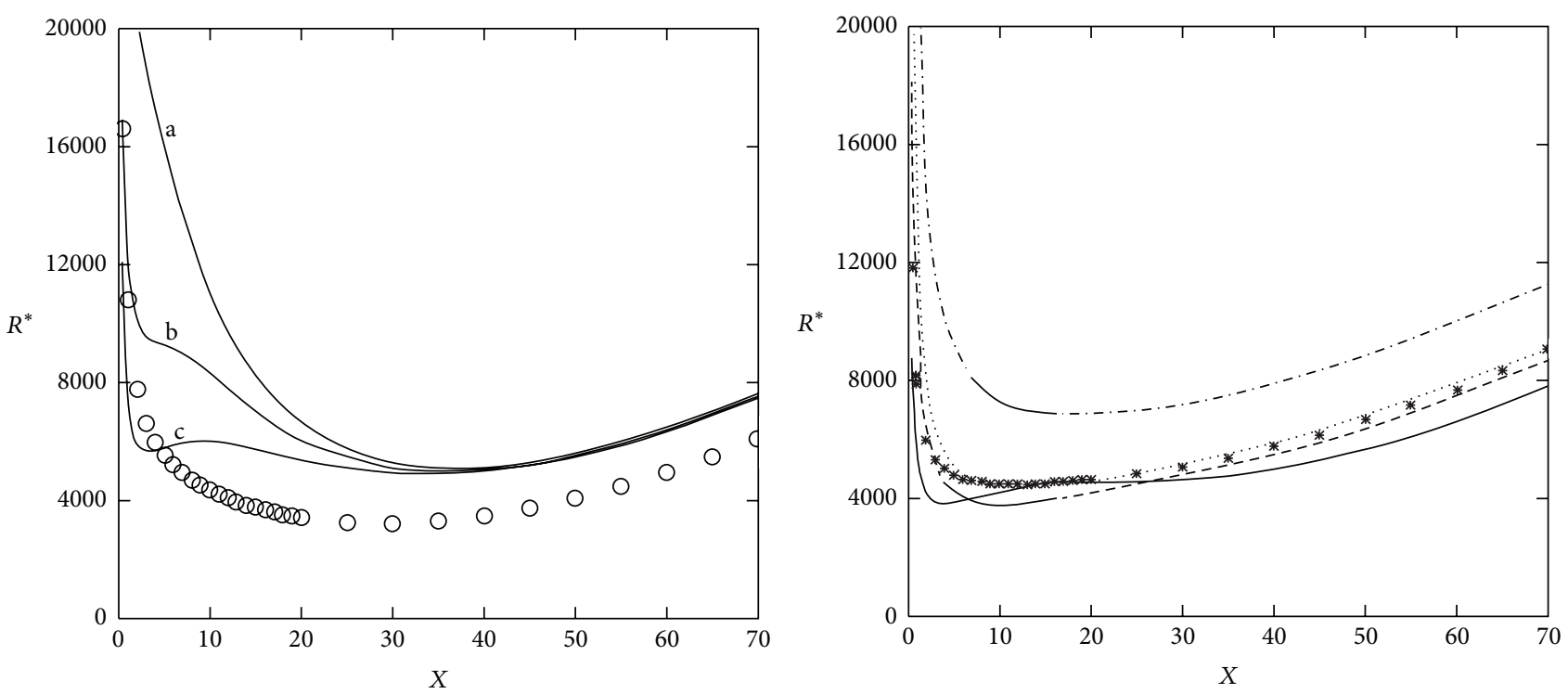

(b)

FIGURE 2: Thermal instability analysis in the presence of a rotation and a magnetic field. Gravity vector parallel to both rotation vector and magnetic field vector. The dependence of the Rayleigh number $R\left(R^{*}=R / 100\right)$ on the wave number $a\left(x=a^{2} / \pi^{2}\right)$ when $T_{1}=10^{5}$. Left panels: $Q_{1}=40$ (curves labelled a), $Q_{1}=100$ (curves labelled b), $Q_{1}=200$ (curves labelled c), circles: pure rotation $T_{1}=10^{5}$ without magnetic field. Right panels: $Q_{1}=400$ (continuous lines), $Q_{1}=1500$ (dashed lines), $Q_{1}=2000$ (dotted lines), $Q_{1}=4000$ (dash-dotted lines), asterisks: pure magnetic field $Q_{1}=2000$, without rotation. Row (a): free boundaries. Row (b): rigid boundaries.

$\left(Q_{1}=4000\right.$, without rotation $)$ is compared with the dashdotted line $\left(Q_{1}=4000, T_{1}=10^{5}\right)$, it is possible to observe that the Coriolis force induces a marginal stabilizing effect. However when the surfaces are rigid (row (b)) and when the curve with asterisks $\left(Q_{1}=4000\right.$, without rotation $)$ is compared with the dash-dotted line $\left(Q_{1}=4000, T_{1}=10^{5}\right)$, we observe that rotation has a significant stabilizing effect. According to the previous discussion we confirm the fact that when $\mathbf{H}$ and $\boldsymbol{\Omega}$ act simultaneously and when both are parallel to the gravity vector, the stabilizing effects have conflicting tendencies. Figure 3 shows the influence of the Coriolis force when the magnetic number is fixed to $Q_{1}=2000$ and when the surfaces are free (left panel) and rigid (right panel). Notice that when the surfaces are free, the Coriolis force when $T_{1}=$ 1000 (curve labelled a) does not produce any effect on the system as compared to that when there is no rotation (see the asterisks curve for $T_{1}=0$ ). However when the surfaces are rigid (right panel), the Coriolis force when $T_{1}=1000$ induces a stabilizing effect as compared with the case without rotation (see the asterisks curve for $T_{1}=0$ ). It is clearly observed that as the modified Taylor number $T_{1}$ acquires the values $5 \times 10^{5}$ and $10^{6}$, the system becomes more stable. 


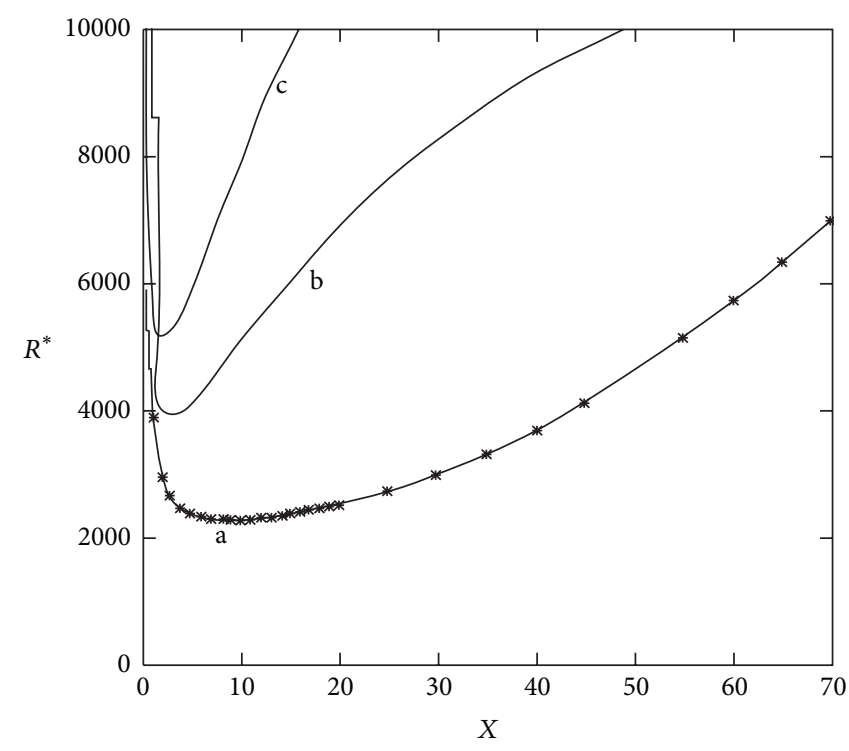

(a)

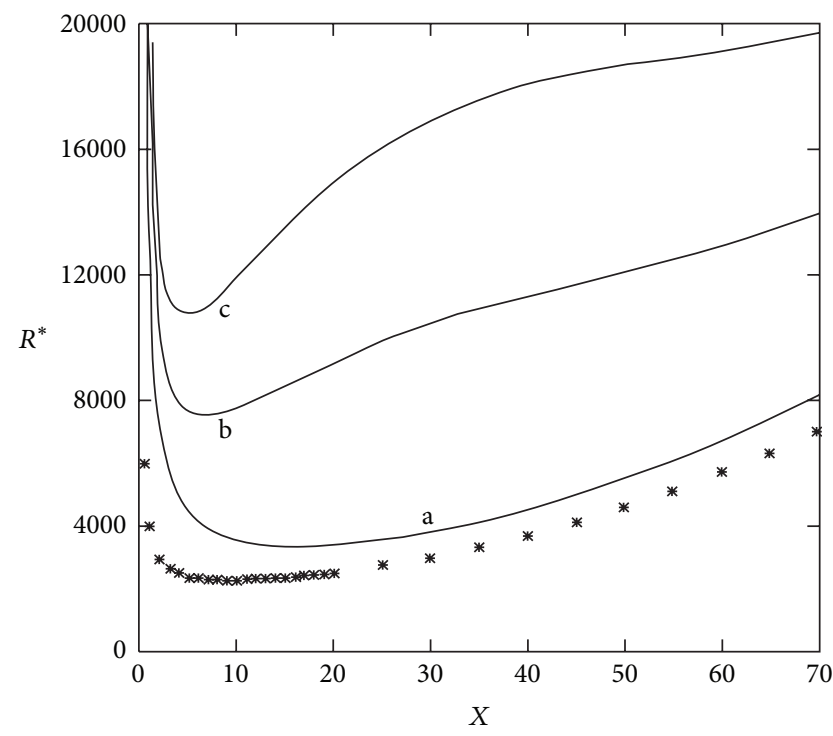

(b)

Figure 3: Thermal instability analysis in the presence of a rotation and a magnetic field. Gravity vector parallel to both rotation vector and magnetic field vector. The dependence of the Rayleigh number $R\left(R^{*}=R / 100\right)$ on the wave number $a\left(x=a^{2} / \pi^{2}\right)$ when $Q_{1}=2000$. The effect of the Coriolis force: $T_{1}=0$ (without rotation, asterisks curves), $T_{1}=1000$ (curves labelled a), $T_{1}=5 \times 10^{5}$ (curves labelled b), and $T_{1}=10^{6}$ (curves labelled c). Left panel: free boundaries. Right panel: rigid boundaries.

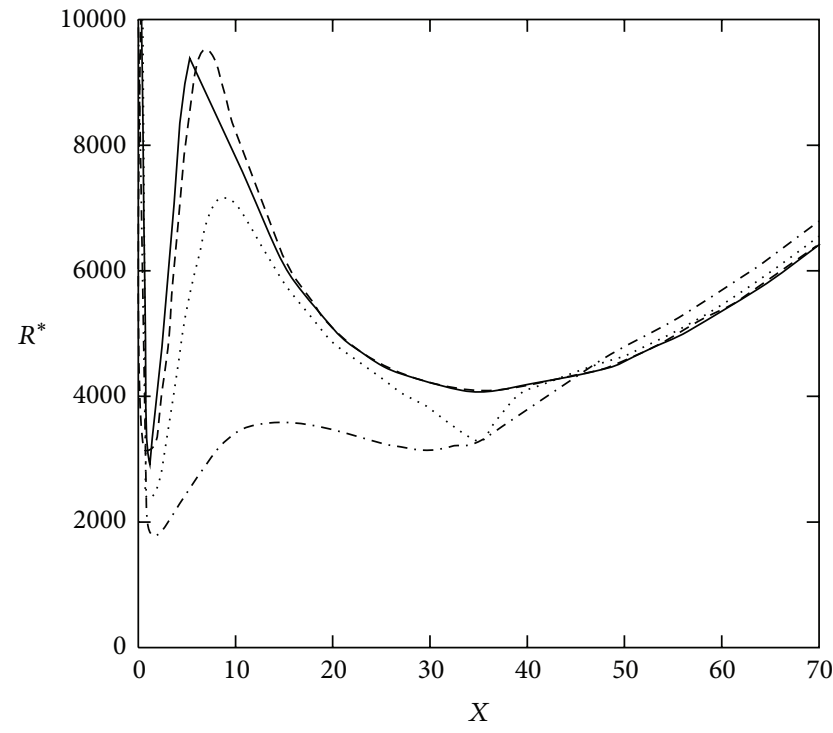

(a)

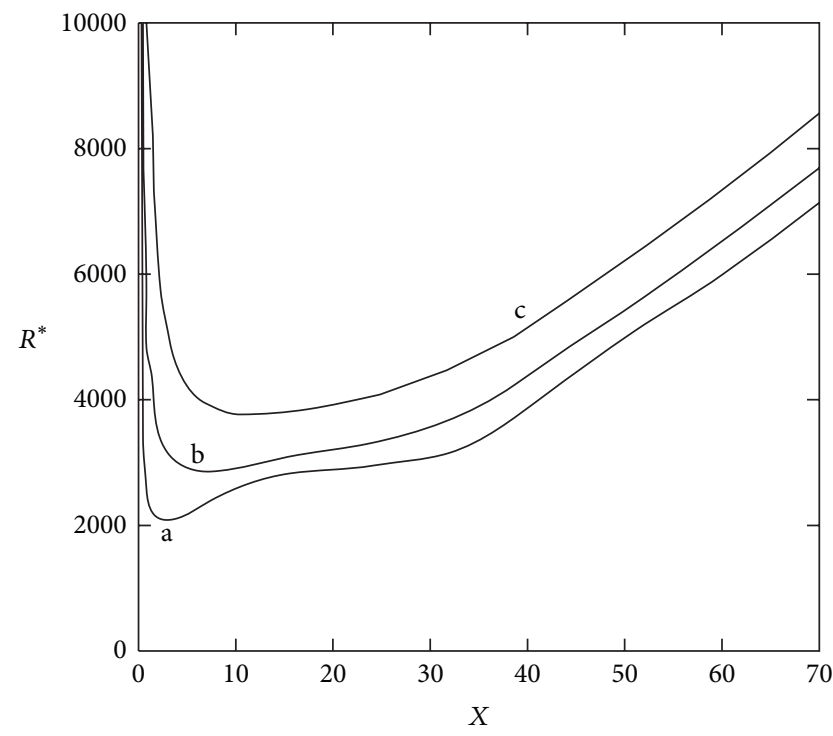

(b)

FIgURE 4: Thermal instability analysis in the presence of a rotation and a magnetic field. Gravity vector parallel to the magnetic field vector $\left(C_{H}=0\right)$. Rotation vector at different values of $C_{R} \neq 0$. The dependence of the Rayleigh number $R\left(R^{*}=R / 100\right)$ for the onset of instability on the wave number $a\left(x=a^{2} / \pi^{2}\right)$ when $T_{1}=10^{5}$ and $Q_{1}=40$ (for free surfaces). $Q_{1}=Q / \pi^{2}$ and $T_{1}=T / \pi^{4}$. Left panel: $C_{R}=0.5$ (continuous line), $C_{R}=1$ (dashed line), $C_{R}=2$ (dotted line), and $C_{R}=4$ (dotted-dashed line). Right panel: $C_{R}=6$ (curve labelled a), $C_{R}=8$ (curve labelled b), and $C_{R}=10$ (curve labelled c).

5.6. Thermal Instability Analysis in the Presence of a Rotation Vector and a Magnetic Field, $\vartheta_{R} \neq 0, \vartheta_{H} \neq 0$. Figure 4 shows, for free surfaces, the dependence of the critical Rayleigh number $R^{*}=R / 100$ and the critical wave number $x=$ $a^{2} / \pi^{2}$, on the parameter $C_{R}$, when the Taylor number
$T_{1}=T / \pi^{4}=10^{5}$, the magnetic parameter $Q_{1}=Q / \pi^{2}=40$, and the gravity vector $\mathbf{g}$ is parallel to the magnetic field vector $\mathbf{H}\left(C_{H}=0\right)$. On the left panel the competition between the stabilizing effect of the Coriolis force and the strong destabilizing properties of the $C_{R}$ parameter when it is in 


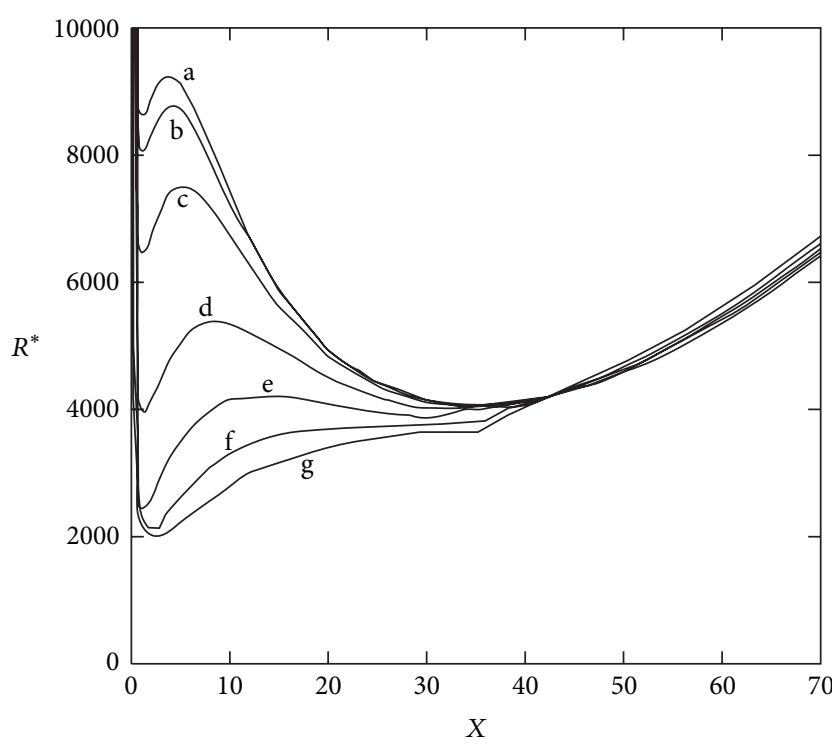

(a)

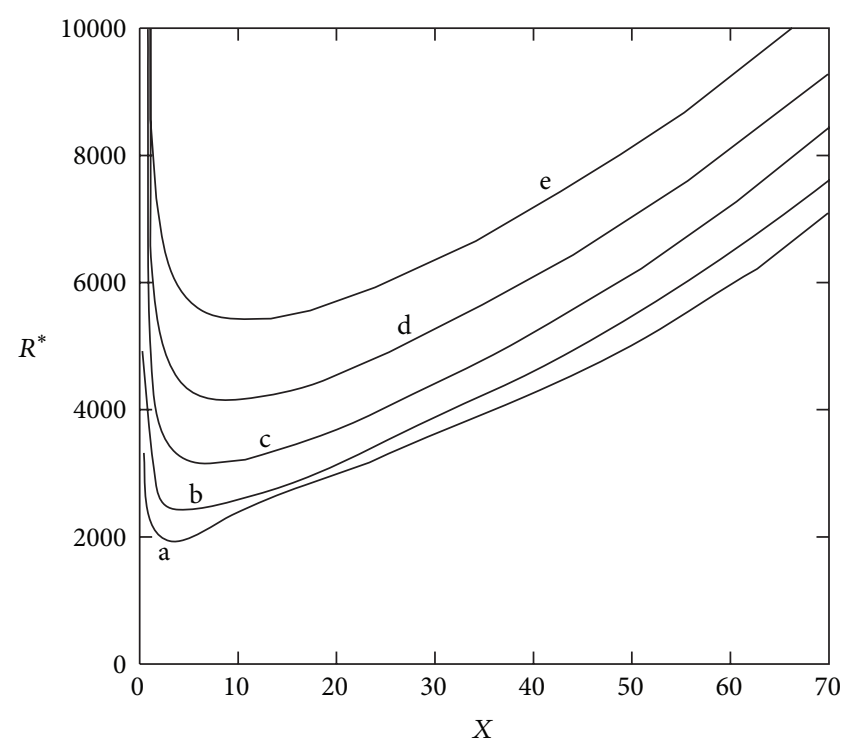

(b)

FIGURE 5: Thermal instability analysis in the presence of a rotation and a magnetic field. Gravity vector parallel to the rotation vector $\left(C_{R}=0\right)$. Magnetic field vector at different values of $C_{H} \neq 0$. The dependence of the Rayleigh number $R^{*}$ for the onset of instability on the wave number $x$ when $T_{1}=10^{5}$ and $Q_{1}=40$ (for free surfaces). Left panel: $C_{H}=0.5$ (curve labelled a), $C_{H}=1$ (curve labelled b), $C_{H}=2($ curve labelled c), $C_{H}=4$ (curve labelled d), $C_{H}=6$ (curve labelled e), $C_{H}=8$ (curve labelled f), and $C_{H}=10$ (curve labelled g). Right panel: $C_{H}=15$ (curve labelled a), $C_{H}=20$ (curve labelled b), $C_{H}=25$ (curve labelled c), $C_{H}=30$ (curve labelled d), and $C_{H}=35$ (curve labelled e).

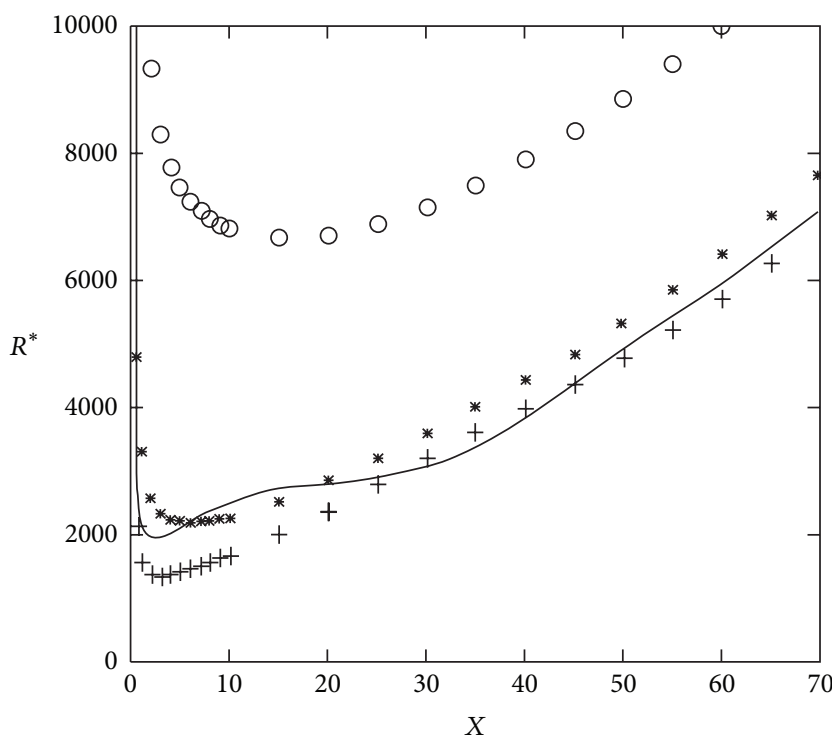

(a)

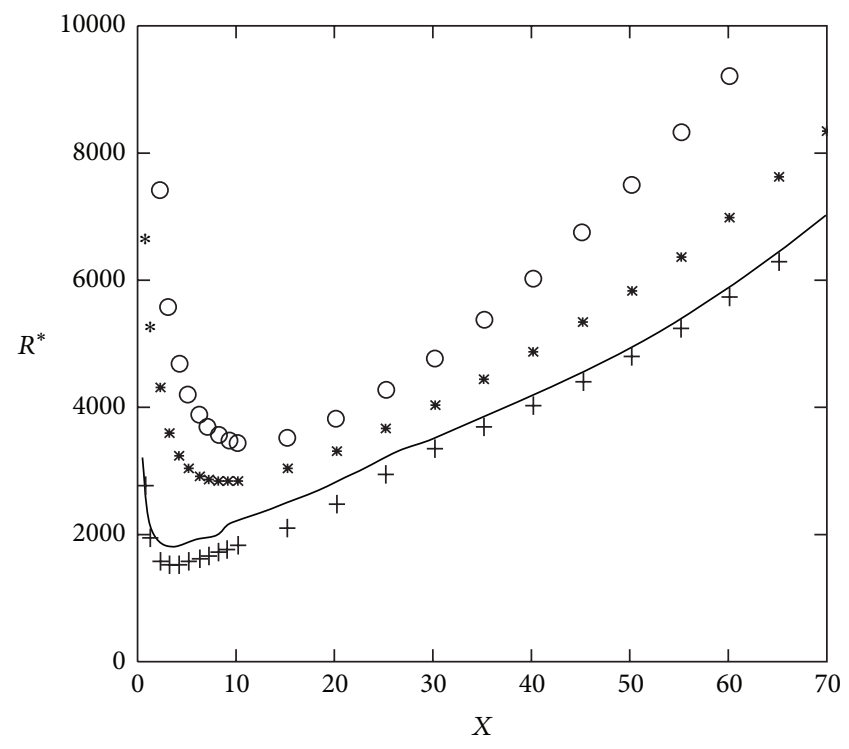

(b)

FIGURE 6: Thermal instability analysis in the presence of a rotation and a magnetic field. The dependence of the Rayleigh number $R^{*}$ for the onset of instability on the wave number $x$ when $T_{1}=10^{5}$ and $Q_{1}=40$ (for free surfaces). Left panel: rotation vector at $C_{R}=6$, for different values of $C_{H}, C_{H}=2$ (continuous line), $C_{H}=10$ ( + curve), $C_{H}=20$ (* curve), and $C_{H}=40$ (o curve). Right panel: magnetic field vector at $C_{H}=15$, for different values of $C_{R}, C_{R}=0.5$ (continuous line), $C_{R}=2$ ( + curve), $C_{R}=10$ (* curve), and $C_{R}=15$ (。 curve).

the range $0.5 \leq C_{R} \leq 4$ is observed. When $C_{R}=0$ and $C_{H}=0$, the critical Rayleigh number is $R^{*}=8829.8$; see left panel of Figure 2, while left panel of Figure 4 shows that when $C_{R}=0.5$, the critical Rayleigh number has been lowered to $R^{*}=3009.2$. Note, on the left panel, the presence of the two minima in the curves of the Rayleigh number $R^{*}$. On the right panel of Figure 4 it is shown that when the $C_{R}$ parameter is in the range $6 \leq C_{R} \leq 10$, the fluid layer becomes more stable as $C_{R}$ increases. Therefore, we have found that there is a critical value of the $C_{R}$ parameter at which the destabilizing 
behaviour is changed to a stabilizing performance, and the critical value occurs around $C_{R}=6$ (see right panel). It is also possible to observe that the curves corresponding to $C_{R}=8$ (curve labelled b) and $C_{R}=10$ (curve labelled c) only show one minimum value. In general it is observed that as the $C_{R}$ parameter increases, the wave number $x=a^{2} / \pi^{2}$ also increases and a tendency to get only one minimum is apparent.

Figure 5 shows, for free surfaces, the dependence of the critical Rayleigh number $R^{*}$ and the critical wave number $x$ on the parameter $C_{H}$, when the Taylor number $T_{1}=10^{5}$, the magnetic parameter $Q_{1}=40$, and the gravity vector $\mathbf{g}$ is parallel to the rotation vector $\Omega\left(C_{R}=0\right)$. On the left panel it is shown that the influence of the parameter $C_{H}$, when its value is 0.5 (curve labelled a), is almost negligible; hence, curve for $C_{H}=0.5$ resembles curve labelled a, of Figure 2 left panel, when $C_{R}=0$ and $C_{H}=0$. On the left panel of Figure 5, it is possible to observe the competition between the stabilizing effect of the Coriolis force and the destabilizing influence of the $C_{H}$ parameter when its values are in the range $0.5 \leq C_{H} \leq 10$; that is, as the $C_{H}$ parameter increases, the fluid layer gets more unstable. Right panel of Figure 5 shows the cooperative behaviour between the Coriolis force and the $C_{H}$ parameter; that is, as the $C_{H}$ parameter increases even more in the range $15 \leq C_{H} \leq 35$, the fluid layer becomes more stable. Hence we have found again that a critical value of the $C_{H}$ parameter exists at which the destabilizing performance of $C_{H}$ is transformed to a stabilizing behaviour. The critical $C_{H}$ is around $C_{H}=15$. We can also conclude that as the $C_{H}$ parameter increases, the wave number $x=a^{2} / \pi^{2}$ also increases and the tendency to get only one minimum is clear.

Finally left panel of Figure 6 shows for free surfaces, the dependence of the Rayleigh number $R^{*}=R / 100$ on the wave number $x=a^{2} / \pi^{2}$ when $C_{R}$ is a fixed value equal to $C_{R}=6$ and $C_{H}$ has the values $C_{H}=2,10,20$, and 40 . Right panel of Figure 6 also shows for free surfaces the dependence of the Rayleigh number $R^{*}$ on the wave number $x$ when $C_{H}$ is a fixed value equal to $C_{H}=15$ and $C_{R}$ has the values $C_{R}=$ $0.5,2,10$, and 15 . In both panels of Figure 6 , the modified Taylor and magnetic numbers are $T_{1}=10^{5}$ and $Q_{1}=40$, respectively. When $C_{R}$ is fixed to $C_{R}=6$ (see left panel), we observe that the curve $C_{H}=2$ (continuous line) resembles the curve $a$ (for $C_{H}=0$ ) shown in right panel of Figure 4 . However the case $C_{H}=2$ is a bit more unstable than the case $C_{H}=0$. When $C_{H}$ increases to $C_{H}=10$ (+ curve), the system becomes even more unstable; however, it is clearly observed that there exists a critical value of $C_{H}$ (around 10), at which the system shifts its unstable behaviour to a more stable performance, see the curves for $C_{H}=20$ (* curve) and $C_{H}=40$ (o curve). When $C_{H}$ is fixed to $C_{H}=15$ (see right panel), we observe that the curve $C_{R}=0.5$ (continuous line) is very similar to the curve $a$ (for $C_{R}=0$ ) shown in Figure 5 right panel. When $C_{R}$ increases to $C_{R}=2$ (+ curve), the system becomes more unstable, however it is clearly observed that there also exists a critical value of $C_{R}$ (around 2), at which the system shifts its unstable behaviour to a more stable performance; see the curves for $C_{R}=10$ (* curve) and $C_{R}=15$ (० curve). In both panels of Figure 6 it is observed that before the critical situation there exists a competition between $C_{H}$ and $C_{R}$ parameters, and the result is that the fluid layer is transformed into a more unstable system. However after the critical situation, there is a cooperative work of $C_{H}$ and $C_{R}$ to inhibit the thermal convection of the infinite layer of fluid.

\section{Conclusions}

The Tau Chebyshev method has been used to solve the classical problem of thermal convection on an infinite fluid layer with rotation and an impressed magnetic field. Our methodology has been verified with results previously published in the literature, through the solution of the first five cases shown in Table 1. By using the Tau Chebyshev method, we find that, for the sixth case shown in Table 1, a critical value of the $C_{R}$ and $C_{H}$ parameters exists at which the competition between $\mathbf{H}$ and $\boldsymbol{\Omega}$, which leads to an unstable system, can be transformed to a cooperative work that promotes a system in which the thermal convection of the layer of fluid is inhibited.

\section{Acknowledgments}

Financial support for this investigation was provided by the Center for Aerospace Research \& Education, University of California, Irvine, CA, USA. R. Avila thanks Professor. Dr. Satya Atluri for his encouragement.

\section{References}

[1] S. Chandrasekhar, Hydrodynamic and Hydromagnetic Stability, Clarendon Press, Oxford, UK, 1961.

[2] S. Chandrasekhar, "The instability of a layer of fluid heated below and subject to Coriolis forces," Proceedings of the Royal Society. London A, vol. 217, pp. 306-327, 1953.

[3] S. Chandrasekhar and D. D. Elbert, "The instability of a layer of fluid heated below and subject to Coriolis forces. II," Proceedings of the Royal Society. London A, vol. 231, pp. 198-210, 1955.

[4] S. Chandrasekhar, "The instability of a layer of fluid heated below and subject to the simultaneous action of a magnetic field and rotation," Proceedings of the Royal Society. London A, vol. 225, pp. 173-184, 1954.

[5] S. Chandrasekhar, "On the inhibition of convection by a magnetic field," The Philosophical Magazine, vol. 43, pp. 501-532, 1952.

[6] B. Lehnert and N. C. Little, "Experiments on the effect of inhomogeneity and obliquity of a magnetic field in inhibiting convection," Tellus, vol. 9, pp. 97-103, 1957.

[7] P. Falsaperla and S. Lombardo, "Competition and cooperation of stabilizing effects in the Bénard problem with Robin type boundary conditions," in Proceedings of the 15th Conference on Waves and Stability in Continuous Media (WASCOM '09), pp. 140-145, World Scientific, 2010.

[8] S. Chandrasekhar, "The instability of a layer of fluid heated below and subject to the simultaneous action of a magnetic field and rotation. II," Proceedings of the Royal Society. London A, vol. 237, pp. 476-484, 1956.

[9] P. Falsaperla, Stability in convection problems for fluids and flows in porous media with general boundary conditions [Ph.D. thesis], Universita degli Studi di Catania, 2010. 
[10] S. Lombardo, "Stability in the Bénard problems with competing effects via the reduction method," in Proceedings of the14th Conference on Waves and Stability in Continuous Media (WASCOM '08), pp. 372-377, World Scientific, 2008.

[11] P. Falsaperla and G. Mulone, "Stability in the rotating Bénard problem with Newton-Robin and fixed heat flux boundary conditions," Mechanics Research Communications, vol. 37, no. 1, pp. 122-128, 2010.

[12] A. S. Orszag, "Accurate solution of the Orr-Sommerfeld stability equation," Journal of Fluid Mechanics, vol. 50, pp. 689-703, 1971.

[13] J. J. Dongarra, B. Straughan, and D. W. Walker, "Chebyshev tauQZ algorithm methods for calculating spectra of hydrodynamic stability problems," Applied Numerical Mathematics, vol. 22, no. 4, pp. 399-434, 1996.

[14] D. Bourne, "Hydrodynamic stability, the Chebyshev tau method and spurious eigenvalues," Continuum Mechanics and Thermodynamics, vol. 15, no. 6, pp. 571-579, 2003.

[15] D. Gottlieb and S. A. Orszag, "Numerical analysis of spectral methods: theory and applications," in CBMS-NSF Regional Conference Series in Applied Mathematics, vol. 26, SIAM, Philadelphia, Pa, USA, 1977.

[16] G. B. McFadden, B. T. Murray, and R. F. Boisvert, "Elimination of spurious eigenvalues in the Chebyshev tau spectral method," Journal of Computational Physics, vol. 91, no. 1, pp. 228-239, 1990. 


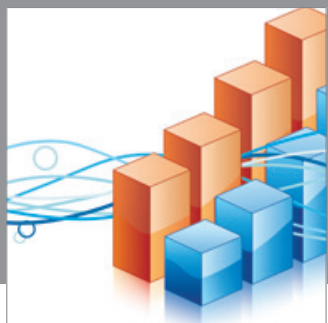

Advances in

Operations Research

mansans

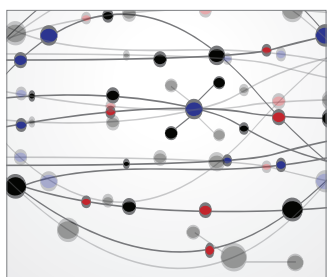

The Scientific World Journal
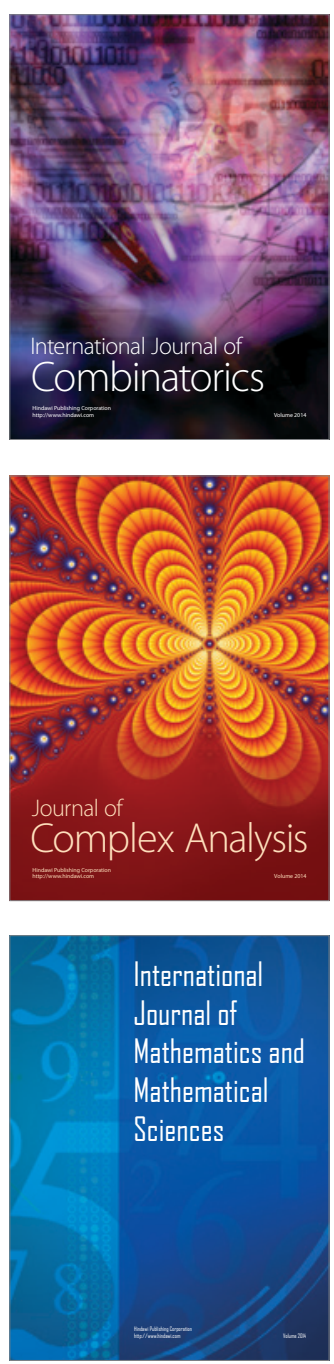
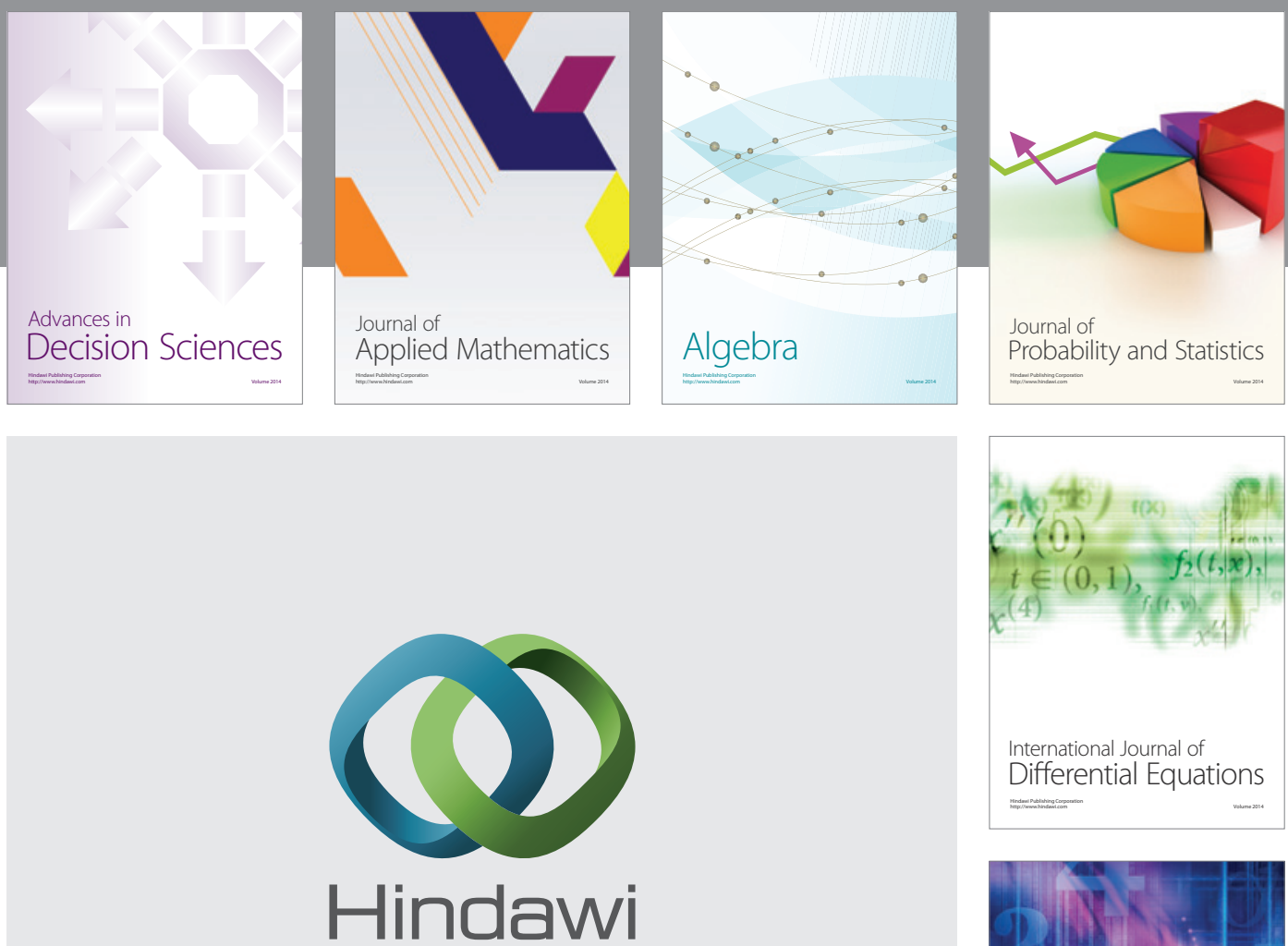

Submit your manuscripts at http://www.hindawi.com
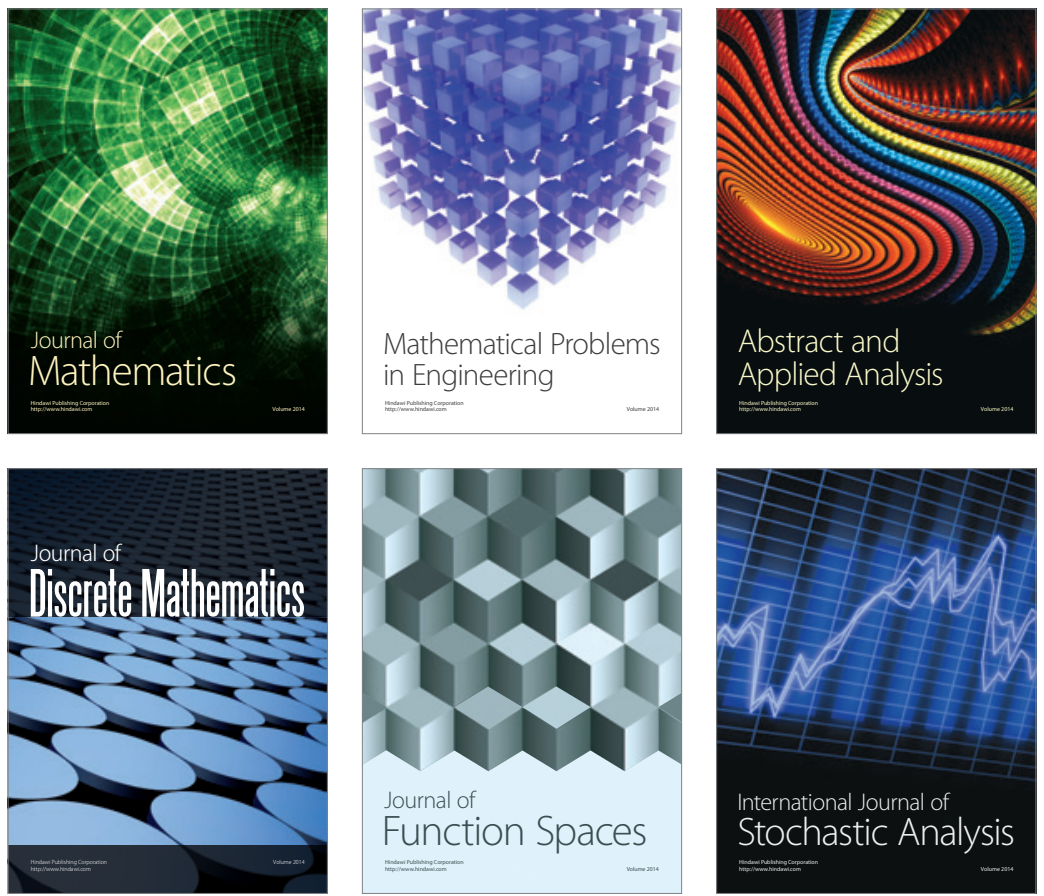

Journal of

Function Spaces

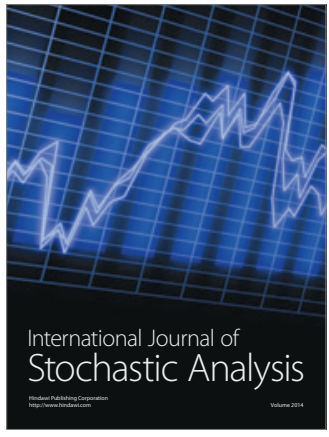

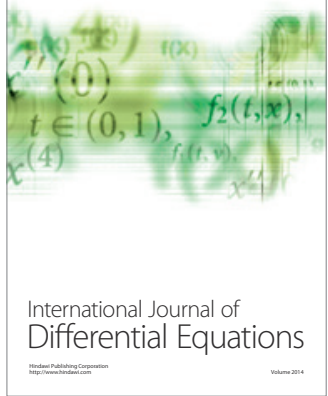
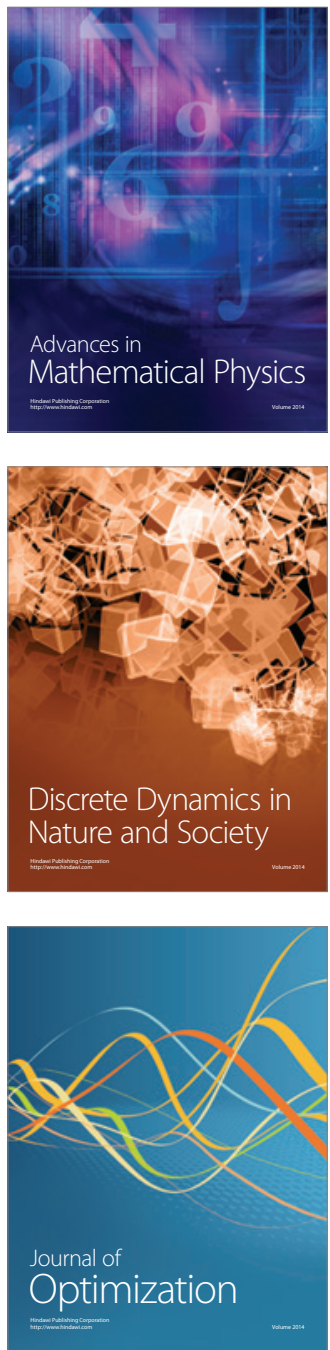